\title{
NEOWISE OBSERVATIONS OF NEAR-EARTH OBJECTS: PRELIMINARY RESULTS
}

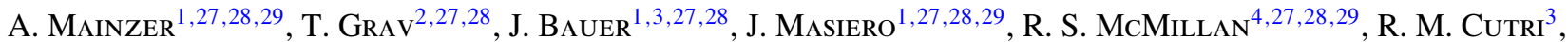

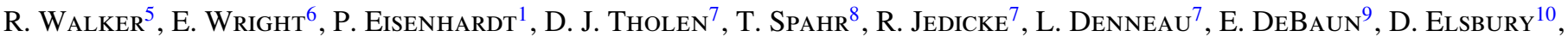 \\ T. Gautier ${ }^{11}$, S. Gomillion ${ }^{12}$, E. Hand ${ }^{13}$, W. Mo ${ }^{2}$, J. WAtKins ${ }^{14}$, A. Wilkins ${ }^{15}$, G. L. Bryngelson ${ }^{16,27,29}$, \\ A. Del Pino Molina ${ }^{17,28}$, S. Desai ${ }^{18,27}$, M. Gómez Camus ${ }^{19,28}$, S. L. Hidalgo ${ }^{17,28}$, I. Konstantopoulos ${ }^{20,27,29}$, \\ J. A. Larsen ${ }^{21,27,28,29}$, C. Maleszewski ${ }^{4,27,28,29}$, M. A. MalKaN ${ }^{6,27,29}$, J.-C. Mauduit ${ }^{22,27,29}$, B. L. Mullan ${ }^{20,28}$, \\ E. W. OlszewsKi ${ }^{23,28}$, J. Pforr ${ }^{24,28}$, A. SARo ${ }^{25,28}$, J. V. Scottit ${ }^{4,27,28,29}$, AND L. H. Wasserman ${ }^{26,28}$ \\ ${ }^{1}$ Jet Propulsion Laboratory, California Institute of Technology, Pasadena, CA 91109 USA; amainzer@jpl.nasa.gov \\ ${ }^{2}$ Department of Physics and Astronomy, Johns Hopkins University, Baltimore, MD, USA \\ ${ }^{3}$ Infrared Processing and Analysis Center, California Institute of Technology, Pasadena, CA 91125, USA \\ ${ }^{4}$ Lunar and Planetary Laboratory, University of Arizona, 1629 East University Blvd., Tucson, AZ 85721-0092, USA \\ ${ }^{5}$ Monterey Institute for Research in Astronomy, Monterey, CA, USA \\ ${ }^{6}$ Department of Physics and Astronomy, UCLA, P.O. Box 91547, Los Angeles, CA 90095-1547, USA \\ ${ }^{7}$ Institute for Astronomy, University of Hawaii, 2680 Woodlawn Drive, Honolulu, HI, USA \\ ${ }^{8}$ Minor Planet Center, Harvard-Smithsonian Center for Astrophysics, 60 Garden Street, Cambridge, MA 02138, USA \\ ${ }^{9}$ Department of Physics and Astronomy, Dartmouth University, Hanover, NH 03755, USA \\ ${ }^{10}$ University of California Santa Barbara, Broida Hall, Santa Barbara, CA 93103, USA \\ ${ }^{11}$ Cornell University, Ithaca, NY 14853, USA \\ ${ }^{12}$ Department of Engineering Physics, Embry-Riddle Aeronautical University, 600 S. Clyde Morris Boulevard, Daytona Beach, FL 32114, USA \\ ${ }^{13}$ Department of Mechanical Engineering, University of Missouri-Kansas City, Kansas City, MO 64110, USA \\ ${ }^{14}$ Department of Earth and Space Sciences, UCLA, 595 Charles Young Drive East, Box 951567, Los Angeles, CA 90095, USA \\ ${ }^{15}$ Department of Astronomy, University of Maryland, College Park, MD 20742, USA \\ ${ }^{16}$ Department of Physics and Astronomy, Clemson University, 8304 University Station, Clemson, SC 29634, USA \\ ${ }^{17}$ Instituto de Astrofisica de Canarias, Vìa Lactea, E38200-La Laguna, Tenerife, Canary Islands, Spain \\ ${ }^{18}$ National Center for Supercomputing Applications, University of Illinois, Urbana-Champagne, 1205 W. Clark St., Urbana, IL 61801, USA \\ ${ }^{19}$ Departmento de Ciencias Fisicas, Facultad de Ingenieria, Universidad Andres Bello, Republica 220, Santiago, Chile \\ ${ }^{20}$ Department of Astronomy and Astrophysics, Penn State University, 525 Davey Lab, University Park, PA 16802, USA \\ ${ }^{21}$ Department of Physics, United States Naval Academy, Annapolis, MD 21402, USA \\ ${ }^{22}$ Infrared Processing and Analysis Center/Spitzer Science Center, California Institute of Technology, Mail Code 220-6, Pasadena, CA 91125, USA \\ ${ }^{23}$ Steward Observatory, University of Arizona, Tucson, AZ 85721, USA \\ ${ }^{24}$ Institute of Cosmology and Gravitation, University of Portsmouth, Dennis Sciama Building, Burnaby Road, Portsmouth PO1 3FX, UK \\ ${ }^{25}$ Department of Physics, Ludwig-Maximilians-Universitat, Scheinerstr. 1, 81679, Munich, Germany \\ ${ }^{26}$ Lowell Observatory, 1400 W. Mars Hill Road, Flagstaff, AZ 86001, USA \\ Received 2011 July 24; accepted 2011 September 6; published 2011 December 2
}

\begin{abstract}
With the NEOWISE portion of the Wide-field Infrared Survey Explorer (WISE) project, we have carried out a highly uniform survey of the near-Earth object (NEO) population at thermal infrared wavelengths ranging from 3 to $22 \mu \mathrm{m}$, allowing us to refine estimates of their numbers, sizes, and albedos. The NEOWISE survey detected NEOs the same way whether they were previously known or not, subject to the availability of ground-based follow-up observations, resulting in the discovery of more than 130 new NEOs. The survey's uniform sensitivity, observing cadence, and image quality have permitted extrapolation of the 428 near-Earth asteroids (NEAs) detected by NEOWISE during the fully cryogenic portion of the WISE mission to the larger population. We find that there are $981 \pm 19$ NEAs larger than $1 \mathrm{~km}$ and 20,500 \pm 3000 NEAs larger than $100 \mathrm{~m}$. We show that the Spaceguard goal of detecting $90 \%$ of all $1 \mathrm{~km}$ NEAs has been met, and that the cumulative size distribution is best represented by a broken power law with a slope of $1.32 \pm 0.14$ below $1.5 \mathrm{~km}$. This power-law slope produces $\sim 13,200 \pm 1900$ NEAs with $D>140 \mathrm{~m}$. Although previous studies predict another break in the cumulative size distribution below $D \sim 50-100 \mathrm{~m}$, resulting in an increase in the number of NEOs in this size range and smaller, we did not detect enough objects to comment on this increase. The overall number for the NEA population between 100 and $1000 \mathrm{~m}$ is lower than previous estimates. The numbers of near-Earth comets and potentially hazardous NEOs will be the subject of future work.
\end{abstract}

Key words: infrared: planetary systems - minor planets, asteroids: general - planets and satellites: general surveys

Online-only material: color figures, machine-readable table

\footnotetext{
${ }^{27}$ Visiting Astronomer, Kitt Peak National Observatory, National Optical Astronomy Observatory, which is operated by the Association of Universities for Research in Astronomy (AURA) under cooperative agreement with the National Science Foundation.

${ }^{28}$ Visiting Astronomer, Cerro Tololo Inter-American Observatory, National Optical Astronomy Observatory, which are operated by the Association of Universities for Research in Astronomy, under contract with the National Science Foundation.

${ }^{29}$ The WIYN Observatory is a joint facility of the University of Wisconsin-Madison, Indiana University, Yale University, and the National Optical Astronomy Observatory.
}

\section{INTRODUCTION}

The near-Earth objects (NEOs) are a population of interest for a wide range of scientific investigations and practical considerations. The NEO population (defined as asteroids or comets with perihelion distances $q \leqslant 1.3 \mathrm{AU}$; http://neo.jpl.nasa.gov/neo/groups.html) is thought to be made up of both asteroids and comets ranging in size from objects tens of kilometers in diameter (Shoemaker 1983) down to dust 
grains. Although nearly $\sim 8000$ NEOs have been discovered to date at all size ranges (http://neo.jpl.nasa.gov/stats), this number represents only a fraction of the total population thought to exist (Bottke et al. 2002). Insights into several fundamental questions in planetary science can be gained from large-scale studies of NEO orbital distributions and physical properties such as diameters and albedos.

\subsection{Origins and Evolution of NEOs}

NEOs are a transitory population, with dynamic lifetimes of $10^{6}$ to $10^{8}$ years (Morbidelli \& Gladman 1998). They must therefore be continually replenished in order to maintain the population we observe today. It has been shown that NEOs are likely to have been delivered primarily from specific regions (Wetherill 1988; Rabinowitz 1997a, 1997b; Bottke et al. 2002) such as the $v_{6}$ secular resonance and 3:1 mean motion resonance with Jupiter. The near-Earth asteroids (NEAs) in the NEO population are thought to have been pushed by the Yarkovsky thermal drift force from different parts of the Main Belt into resonances that may preferentially inject them into the inner solar system (Bottke et al. 2002). Near-Earth comets (NECs) also constitute a part of the NEO population and are thought to originate from a variety of regions, including the Kuiper Belt, the scattered disk, the Oort cloud, and the Jupiter Trojan clouds (Levison \& Duncan 1997; Duncan \& Levison 1997; Weissman 1996; Levison et al. 1997). Efforts to more precisely constrain the origins of the NEOs have been hampered by the relative paucity of observational data. Surveys such as the Lincoln NearEarth Asteroid Research Program, the Near-Earth Asteroid Tracking Program, the Lowell Near-Earth Object Survey, the Catalina Sky Survey, PanSTARRS, and Spacewatch (Stokes et al. 2000; Helin et al. 1997; Koehn \& Bowell 2000; Larson 2007; McMillan 2007) have identified NEOs, but they observe in visible wavelengths, so they are not able to sense low albedo NEOs as effectively nor can visible light measurements provide strong constraints on diameters, albedos, or other physical characteristics. Size and albedo distributions for NEOs derived from these visible light surveys remain uncertain (particularly for sub-kilometer objects) since these models rely upon the absolute magnitude $(H)$ through an assumed albedo to derive size (Stuart \& Binzel 2004; Morbidelli et al. 2002; Shoemaker et al. 1990; Luu \& Jewitt 1998). Studies linking meteorite falls to NEO source regions in the main asteroid belt have been performed (e.g., Thomas \& Binzel 2010; Vernazza et al. 2008), but meteorites have been heavily processed by passage through Earth's atmosphere, which also introduces biases in composition and size.

Bottke et al. (2002) were limited in their ability to model the NEO population by the fact that the Spacewatch data upon which their results were based provided them only a very small sample of optically selected NEOs. The paucity of physical characterization data for large numbers of NEOs has complicated efforts to identify weaker source regions. Models of NEO origins such as Bottke et al. (2002) can be tested and improved by obtaining a sample of NEOs with accurately measured diameters and albedos, well-known orbits, and well-understood survey detection biases and efficiency. Infrared observations of all classes of minor planets are useful for determining size and albedo distributions, as well as thermophysical properties such as thermal inertia, the magnitude of non-gravitational forces, and surface roughness (e.g., Bhattacharya et al. 2010; Tedesco et al. 2002; Trilling et al. 2010; Harris et al. 2009).

\subsection{NEOs As Impactors}

Asteroids and comets have impacted Earth throughout its history, profoundly altering the course of life on Earth (Alvarez et al. 1980; Hildebrand et al. 1991). Currently available estimates of the size distribution of potentially hazardous NEOs lead to the assessment that objects capable of causing global catastrophe (larger than $1 \mathrm{~km}$ in diameter) are thought to impact approximately every 700,000 years (National Research Council 2010). However, smaller objects can still cause considerable damage (NEO Science Definition Team 2003; Chapman 2004). Recent simulations suggest that the 1908 Tunguska impact could have been caused by an object as small as 30-50 m (National Research Council 2010), which would imply that the potential for damage caused by smaller objects has been underestimated. However, this increased risk from smaller impacts is offset by recent population estimates (Boslough \& Harris 2008; Harris 2008) that indicate that the population of asteroids in the size range between several tens to hundreds of meters in diameter may be as much as a factor of three less than estimated using a straight-line power law of slope -2.354 as proposed by Bottke et al. (2002) or a slope of -1.87 . Nevertheless, considerable uncertainty about the true NEO size distribution persists due to the lack of accurate diameters, albedos, and surveys that are not substantially biased against low albedo objects. Even less is known about the subset of NEOs that are considered potentially hazardous.

\section{OBSERVATIONS}

Wide-field Infrared Survey Explorer (WISE) is a NASA Medium-class Explorer mission designed to survey the entire sky in four infrared wavelengths, 3.4, 4.6, 12, and $22 \mu \mathrm{m}$ (denoted $W 1, W 2, W 3$, and $W 4$, respectively). In a Sunsynchronous orbit, WISE observed the entire sky in all four filters simultaneously using three beamsplitters and a scan mirror. The pre-launch description of the mission, including its design and construction, is given in Liu et al. (2008) and Mainzer et al. (2005), and the post-launch performance is discussed in Wright et al. (2010). The final mission data products are a multi-epoch image atlas and source catalogs that will serve as an important legacy for future research. The survey has yielded observations of over 157,000 minor planets, including NEOs, Main Belt Asteroids (MBAs), comets, Hildas, Trojans, Centaurs, and scattered disk objects (Mainzer et al. 2011a, hereafter M11A). WISE has observed nearly two orders of magnitude more minor planets than its predecessor, the Infrared Astronomical Satellite (IRAS; Tedesco et al. 1988; Matson 1986). WISE was launched on 2009 December 14. The WISE survey began on 2010 January 14, and the mission exhausted its primary tank cryogen on 2010 August 5. An augmentation to the WISE baseline data processing pipeline, "NEOWISE," permitted a search for new moving objects to be carried out using the WISE data in near-real time. NEOWISE also allowed the individual exposures for each part of the sky (an average of eight on the ecliptic plane, but rising into the hundreds near the ecliptic poles) to be archived and made available to the public. Exhaustion of the secondary cryogen tank occurred on 2010 October 1, and the survey was continued as the NEOWISE Post-Cryogenic Mission using only bands $W 1$ and $W 2$ until 2011 February 1.

As described in Wright et al. (2010) and M11A, the NEOWISE survey cadence resulted in most minor planets in the WISE sample receiving an average of 10-12 observations 


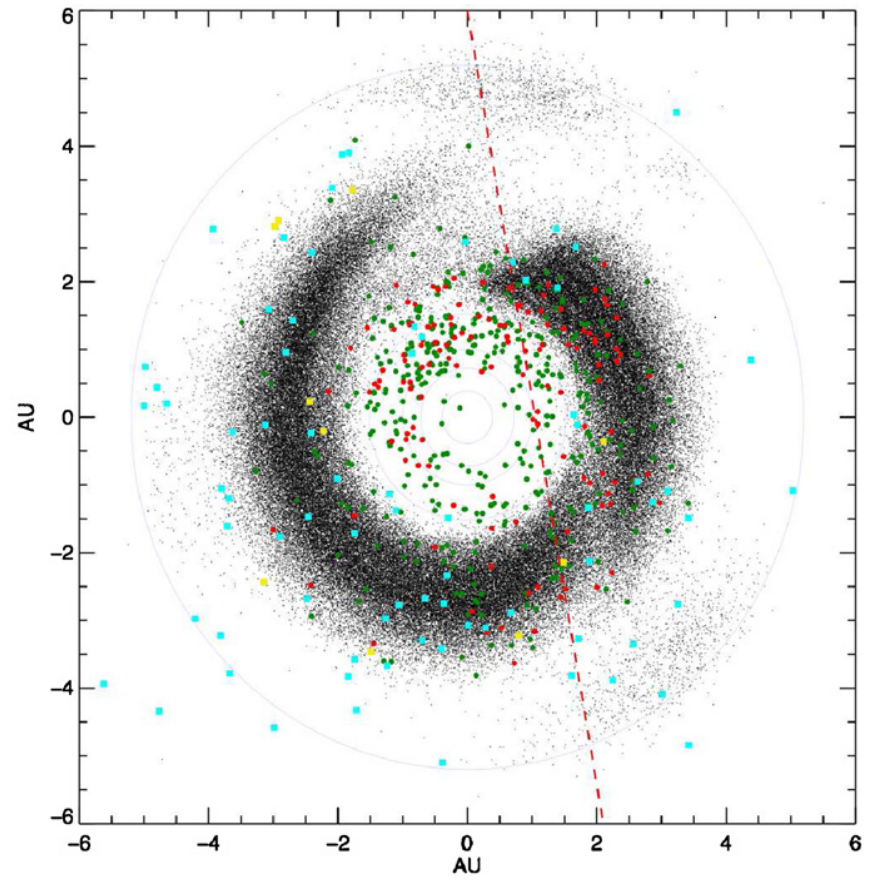

Figure 1. WISE always surveys near $90^{\circ}$ solar elongation; this figure shows a top-down view of the objects detected by NEOWISE as of 2011 February (distances are given in AU). The outermost circle represents Jupiter's orbit; the interior circles represent the terrestrial planets. Previously known NEOs are shown as green circles; new NEOs discovered by NEOWISE are shown as red circles; previously known comets observed by WISE are shown as cyan squares, and comets discovered by NEOWISE are shown as yellow squares. All other objects are shown as black points. The drop in density of objects observed near $(+2,+2) \mathrm{AU}$ in the figure is due to the exhaustion of the secondary tank's cryogen on 2010 August 5, resulting in the loss of band W4. The dashed red line indicates the survey scan plane at the time of the exhaustion of the primary tank and the start of the NEOWISE Post-Cryogenic Mission on 2010 October 1; the survey was completed on 2011 February 1 . The drop in detections near $(+2$, -2) $\mathrm{AU}$ in the figure is due to the intersection of the galactic plane with the ecliptic plane; the higher backgrounds and confusion caused by galactic cirrus resulted in the identification of fewer sources.

(A color version of this figure is available in the online journal.)

over $\sim 36 \mathrm{hr}$, although some NEOs were observed dozens or even hundreds of times. NEOWISE detected minor planets using the WISE Moving Object Processing System (WMOPS). WMOPS was adapted from the Pan-STARRS Moving Object Processing System (Kubica et al. 2007) to account for the different cadence and observing strategy used by WISE. During the entire period of survey operations, NEOWISE is known to have observed at least 584 NEOs and is responsible for the discovery of 135 new, previously unknown NEOs. For purposes of determining the debiased population characteristics of the NEOs, in this paper we consider only those objects detected during the fully cryogenic portion of the survey by the WMOPS pipeline using the first-pass WISE data processing pipeline as described below. This sample consists of a set of 428 NEOs, of which 314 were recoveries of objects discovered by other groups, and 114 were NEOWISE discoveries. By considering this subset of NEOs, we can study a population that was observed in a more-or-less uniform fashion. This approach facilitates the understanding of the NEOWISE survey biases, allowing us to model the unobserved portion of the NEO population. We will consider the NEOs detected during the post-cryogenic portion of the survey in a future work. Figure 1 shows the objects detected by NEOWISE using the first-pass data processing pipeline; while most of the solar system was surveyed during the fully cryogenic portion of the mission, some of it was observed with only bands $W 1$ and $W 2$, leading to a significant drop in sensitivity. For this and other reasons, we must debias the survey in order to understand the properties of the NEO population and disentangle them from survey biases.

The observations of the objects listed in Table 1 were retrieved by querying the Minor Planet Center's (MPC) observation files to look for all instances of individual WISE detections of the desired objects that were reported using the WMOPS. The resulting set of position/time pairs were used as the basis of a query of WISE source detections processed with the First Pass version of the WISE data process pipeline (Version 3.5) in individual exposures (also known as "Level 1b" images) using the Infrared Science Archive (IRSA; Cutri et al. 2011). In order to ensure that only observations of the desired moving object were returned from the query, the search radius was restricted to 0.3 arcsec from the position listed in the MPC observation file. A radius of 0.3 arcsec was chosen to account for any round-off error between the decimal format used by the WISE catalog and the hexigesimal format used by the MPC. Additionally, since WISE collected a single exposure every $11 \mathrm{~s}$, the modified Julian date was required to be within $2 \mathrm{~s}$ of the time specified by the MPC. The artifact identification flag cc_flags was allowed to be equal to either $0, \mathrm{P}$, or $\mathrm{p}$; this flag indicates that the sources were unlikely to have been affected by persistence. We required that the flag ph_qual be equal to A, B, or C (this flag indicates that the source is likely to have been a valid detection). As described in Mainzer et al. (2011b, hereafter M11B) and Cutri et al. (2011), we included observations with magnitudes close to experimentally derived saturation limits, but when sources became brighter than $W 1=6, W 2=6, W 3=4$, and $W 4=0$, we increased the error bars on these points to 0.2 mag and applied a linear correction to W3 (see the WISE Explanatory Supplement for details). Each object had to be observed a minimum of three times in at least one WISE band, and to avoid having low-level noise detections and/or cosmic rays contaminating our thermal model fits, we required that observations in more than one band appear at least $40 \%$ of the number of observations found in the band with the largest number of observations (usually $W 2$ or $W 3$ for NEOs). The WMOPS system is designed to reject inertially fixed objects such as stars and galaxies in bands $W 3$ and $W 4$. However, the source density in bands $W 1$ and $W 2$ is $\sim 100$ times higher than in bands $W 3$ and $W 4$, so it was more likely that asteroid detections were confused with stars or galaxies at these wavelengths. In order to remove such confused asteroid detections, we cross-correlated the individual Level $1 \mathrm{~b}$ detections with the WISE atlas and daily co-add catalogs. Objects within 6.5 arcsec (equivalent to the WISE beam size at bands $W 1, W 2$, and $W 3$ ) of the asteroid position which appeared in the co-added source lists at least twice and which appeared more than $30 \%$ of the total number of coverages of a given area of sky were considered to be inertially fixed sources; these asteroid detections were considered contaminated and were not used for thermal fitting.

During the cryogenic portion of the survey, 231 objects were placed on the MPC's NEO Confirmation Page (http://www.minorplanetcenter.org/iau/NEO/ToConfirm.html). Of these, 115 were designated as NEOs, while the remaining candidates that received visible light follow-up were confirmed to be MBAs, Trojans, etc. (of the $\sim 300,000$ tracklets submitted to the MPC, only a handful were rejected as spurious). Follow-up observations of NEOWISE-discovered NEOs were carried out by a world-wide network of amateur and 


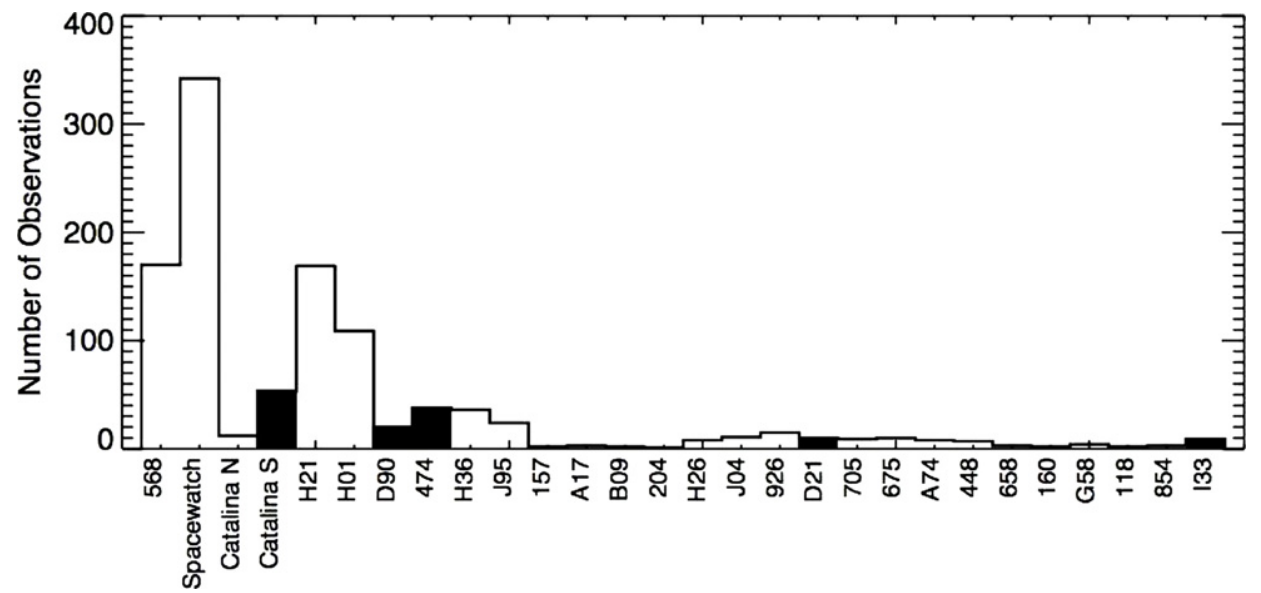

Figure 2. Follow-up observations within $\sim 2$ weeks of the last observations of NEO candidates detected by NEOWISE were essential for securing an orbit, as the average NEOWISE arc spanned $\sim 36 \mathrm{hr}$. After 2 weeks, most objects detected with such arcs have sufficiently high astrometric error that they cannot be recovered. This figure shows the number of follow-up observations contributed within 15 days of a newly discovered NEO's last observation by NEOWISE as a function of MPC observatory code. Spacewatch (codes 291, 691, 695, and 807), H21 (the Killer Asteroid Project; http://killerasteroidproject.org/student_obs.htm), 568 (Mauna Kea, observers D. Tholen, and students), and H01 (Magdalena Ridge Observatory; Ryan \& Ryan 2008) were the most prolific contributors within the first 15 days; other observers contributed follow-up over longer time spans. Observatories in the southern hemisphere are shaded black, illustrating the paucity of southern facilities that were available for follow-up of NEOWISE discoveries. In spite of all of the follow-up observations shown in this figure, 15 NEOWISE-discovered NEOs were designated without any visible follow-up, and another $\sim 15-20$ objects appeared on the MPC Confirmation Page as probable NEOs but received neither designations nor follow-up; these objects were lost.

Table 1

NEATM Results for the 428 NEOs Detected by NEOWISE During the Fully Cryogenic Portion of the WISE Mission

\begin{tabular}{|c|c|c|c|c|c|c|c|c|c|c|}
\hline Object & $H$ & $G$ & $\begin{array}{c}D \\
(\mathrm{~km})\end{array}$ & $p_{V}$ & $\eta$ & $p_{\text {IR }}$ & $N(\mathrm{~W} 1)$ & $N(\mathrm{~W} 2)$ & $N(\mathrm{~W} 3)$ & $N(\mathrm{~W} 4)$ \\
\hline K07X10C & 19.40 & 0.15 & $1.047 \pm 0.198$ & $0.028 \pm 0.014$ & $1.350 \pm 0.471$ & $0.045 \pm 0.022$ & 0 & 0 & 13 & 0 \\
\hline K10HA8Z & 21.00 & 0.15 & $0.356 \pm 0.067$ & $0.055 \pm 0.032$ & $1.350 \pm 0.475$ & $0.089 \pm 0.051$ & 0 & 0 & 11 & 0 \\
\hline 85713 & 15.70 & 0.15 & $3.484 \pm 0.789$ & $0.076 \pm 0.039$ & $1.350 \pm 0.450$ & $0.122 \pm 0.062$ & 0 & 0 & 9 & 0 \\
\hline 07822 & 17.40 & 0.15 & $1.208 \pm 0.015$ & $0.133 \pm 0.022$ & $2.261 \pm 0.049$ & $0.299 \pm 0.219$ & 23 & 24 & 24 & 24 \\
\hline 07822 & 17.40 & 0.15 & $1.602 \pm 0.012$ & $0.075 \pm 0.014$ & $2.171 \pm 0.030$ & $0.139 \pm 0.043$ & 9 & 10 & 11 & 11 \\
\hline K02N16W & 18.00 & 0.15 & $0.846 \pm 0.009$ & $0.156 \pm 0.033$ & $2.118 \pm 0.066$ & $0.262 \pm 0.156$ & 30 & 40 & 40 & 40 \\
\hline K09V24O & 19.80 & 0.15 & $0.467 \pm 0.016$ & $0.098 \pm 0.020$ & $1.964 \pm 0.130$ & $0.157 \pm 0.167$ & 0 & 5 & 5 & 5 \\
\hline K10MB2U & 20.60 & 0.15 & $0.599 \pm 0.022$ & $0.028 \pm 0.006$ & $1.684 \pm 0.111$ & $0.045 \pm 0.134$ & 0 & 7 & 7 & 7 \\
\hline F4029 & 16.40 & 0.15 & $2.231 \pm 0.050$ & $0.098 \pm 0.021$ & $2.044 \pm 0.085$ & $0.379 \pm 0.084$ & 5 & 5 & 5 & 4 \\
\hline F4029 & 16.40 & 0.15 & $2.164 \pm 0.228$ & $0.104 \pm 0.028$ & $1.396 \pm 0.261$ & $0.166 \pm 0.045$ & 0 & 0 & 12 & 10 \\
\hline 66251 & 17.00 & 0.15 & $1.222 \pm 0.233$ & $0.187 \pm 0.094$ & $1.350 \pm 0.419$ & $0.300 \pm 0.150$ & 0 & 0 & 4 & 0 \\
\hline
\end{tabular}

Notes. This table contains the preliminary thermal fit results based on the First Pass version of the WISE data processing as described in the text. The NEOWISE project plans to produce an updated final catalog of physical properties based on the Second Pass processing of the WISE data using the updated version of the WISE Science Data System, with a goal of delivering this updated catalog to NASA's Planetary Data System. Error values presented here represent the statistical errors on the model fits, including Monte Carlo modeling of uncertainties for the WISE magnitudes, $H, G$, and beaming and $p_{\text {IR }}$ when these two parameters cannot be fit. Two calibration papers (Mainzer et al. 2011b, 2011c) discuss the absolute calibration of the WISE data for small solar system bodies and should be consulted before comparing with data derived from other sources. The quoted precision for each parameter follows the object with the most significant figures for the error on that value in the table. $H, G$, and albedo values of nan or " -9.99 " indicate that the objects have not received visible light follow-up. Beaming value errors of nan or "-9.99" indicate that the thermal fit routine returned a maximum value of pi or a minimum of 0.3 , so error cannot be properly determined. Readers are encouraged to check the WISE Explanatory Supplement (Cutri et al. 2011) for details and updates.

(This table is available in its entirety in a machine-readable form in the online journal. A portion is shown here for guidance regarding its form and content.)

professional astronomers. Figure 2 lists the numbers of observations of NEOWISE discoveries contributed by MPC observatory code within 15 days of the first observation by NEOWISE. Follow-up observations were critically important for securing good long-term orbits of NEOWISE-discovered NEOs because in general, the arc lengths spanned $\sim 36 \mathrm{hr}$. Of the 115 NEOs discovered during the cryogenic mission, 12 received designations despite having no optical follow-up. An additional 22 objects were deemed lost because they received no optical follow-up, and their arcs were too short to designate. The loss of these objects will contribute some uncertainty to the size and albedo distributions computed for NEOs from NEOWISE data; this will be discussed further below.
Figures 3 shows the typical WISE colors for NEOs, MBAs, and Trojan asteroids observed during the cryogenic NEOWISE mission. The NEOs occupy a distinct region of color space and can readily be distinguished from other sources such as stars, distant galaxies, and cool brown dwarfs. Examination of the WISE colors can be used as a means to constrain orbits in cases where an object's orbit is uncertain (e.g., because it has only a short observational arc). Bhattacharya et al. (2010) suggest that thermal colors can be used to identify new candidate minor planets; however, the vast size of the WISE single-exposure source lists (which include transient artifacts such as cosmic rays and latent images) makes techniques that focus on repeated detections of moving sources the preferred method for discovery 

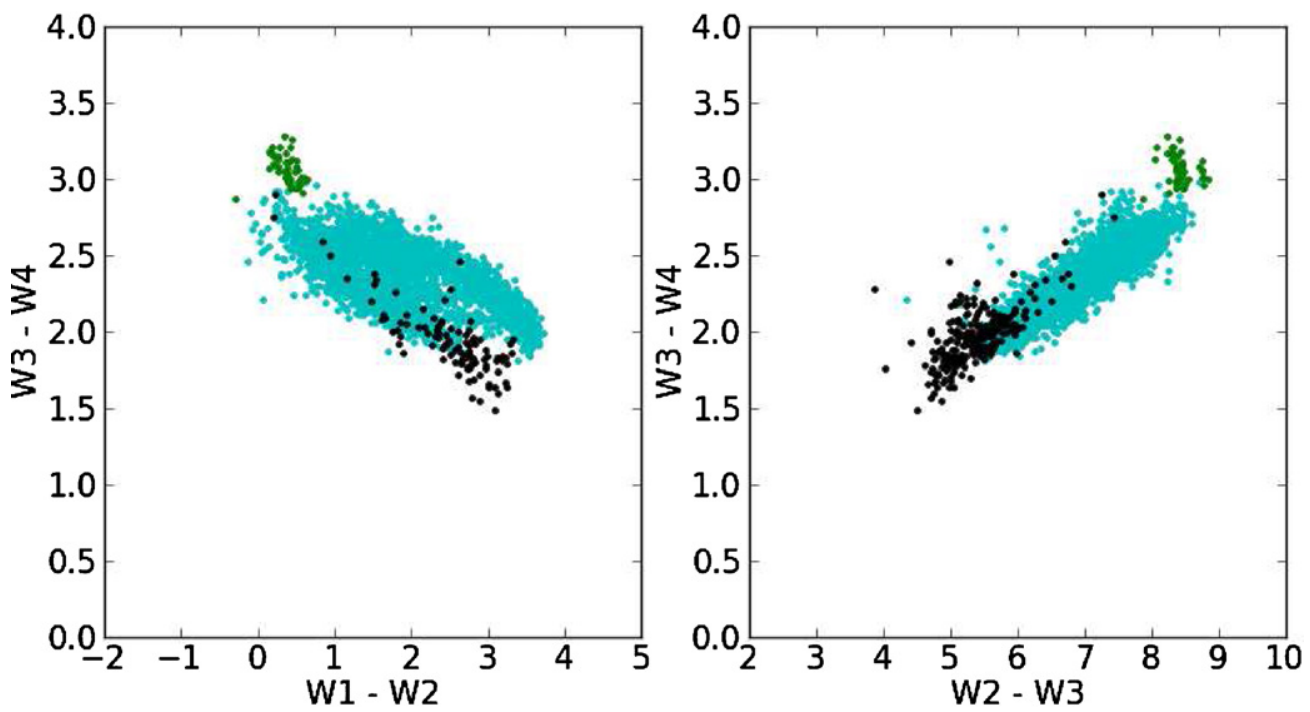

Figure 3. Left: WISE colors for objects observed in bands $W 1, W 2, W 3$, and $W 4$. Black dots are NEOs, cyan dots are Main Belt asteroids, and green dots are Trojan asteroids. Right: WISE colors for NEOs observed in bands $W 1, W 2, W 3$, and $W 4$.

(A color version of this figure is available in the online journal.)
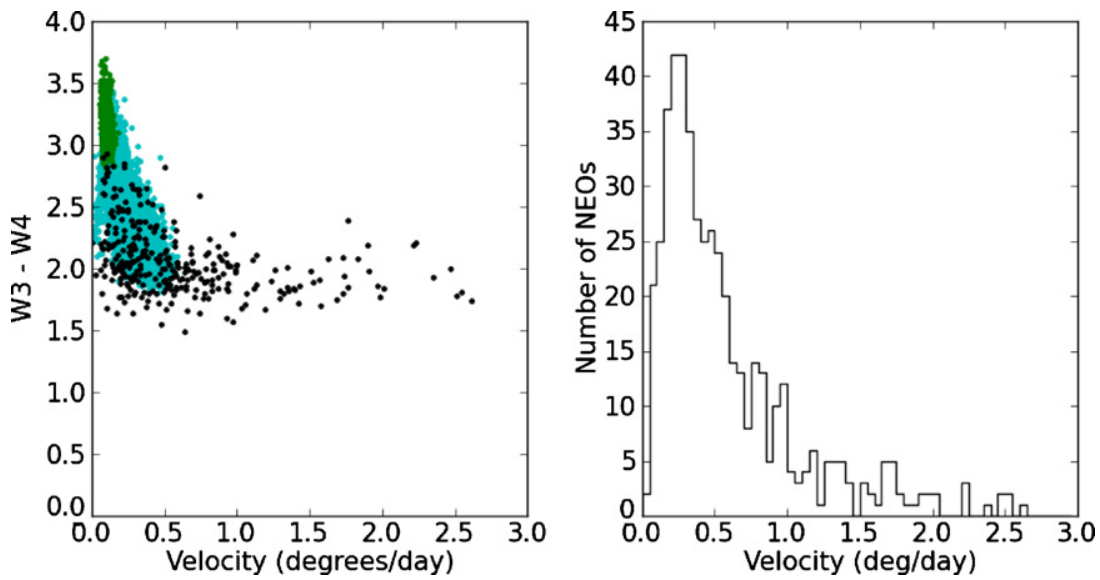

Figure 4. Left: W3-W4 color vs. average sky plane velocity for NEOs detected by NEOWISE. Right: histogram of velocities for NEOs detected by NEOWISE. Black dots are NEOs, cyan dots are Main Belt asteroids, and green dots are Trojan asteroids.

(A color version of this figure is available in the online journal.)

of new objects. Figure 4 shows $W 3-W 4$ versus apparent skyplane velocity and the histogram of sky-plane velocities for observed NEOs; most NEOs detected by NEOWISE are moving at $\sim 0.5$ day $^{-1}$. Figure 5 shows the WISE colors compared with heliocentric distance; as expected, the colors redden as the objects are located at greater distance from the Sun. It may be possible to use the WISE colors and apparent sky-plane velocities to constrain orbits in cases where observational arcs are short and orbits are poorly known. A future work will explore this further.

\section{PRELIMINARY THERMAL MODELING OF NEOs}

We have created preliminary thermal models for each NEO observed by WMOPS during the fully cryogenic portion of the survey with the First Pass version of the WISE data processing pipeline described above; these thermal models will be recomputed when the final data processing is completed. As described in M11B, we employ the spherical near-Earth asteroid thermal model (NEATM) (Harris 1998). The NEATM model uses the so-called beaming parameter $\eta$ to account for cases intermediate between zero thermal inertia, the Standard
Thermal Model (STM) of Lebofsky \& Spencer (1989) and high thermal inertia, the Fast Rotating Model (FRM; Lebofsky et al. 1978; Veeder et al. 1989; Lebofsky \& Spencer 1989). In the STM, $\eta$ is set to 0.756 to match the occultation diameters of (1) Ceres and (2) Pallas. In the FRM, $\eta$ is equal to $\pi$, and the temperature distribution is different than that used in NEATM, being independent of longitude. With NEATM, $\eta$ is a free parameter that can be fit when two or more infrared bands are available (or with only one infrared band if diameter or albedo are known a priori as is the case for objects that have been imaged by visiting spacecraft or observed with radar).

Each object was modeled as a set of triangular facets covering a spherical surface with a variable diameter (cf. Kaasalainen et al. 2004). Although many (if not most) NEOs are nonspherical, the WISE observations generally consisted of $\sim 10-12$ observations per object uniformly distributed over $\sim 36 \mathrm{hr}$ (M11A), so on average, a wide range of rotational phases were sampled. Even though this sampling helps to average out the effects of a rotating non-spherical object, caution must be exercised when interpreting the meaning of an effective diameter in these cases. Figure 6 shows the average 

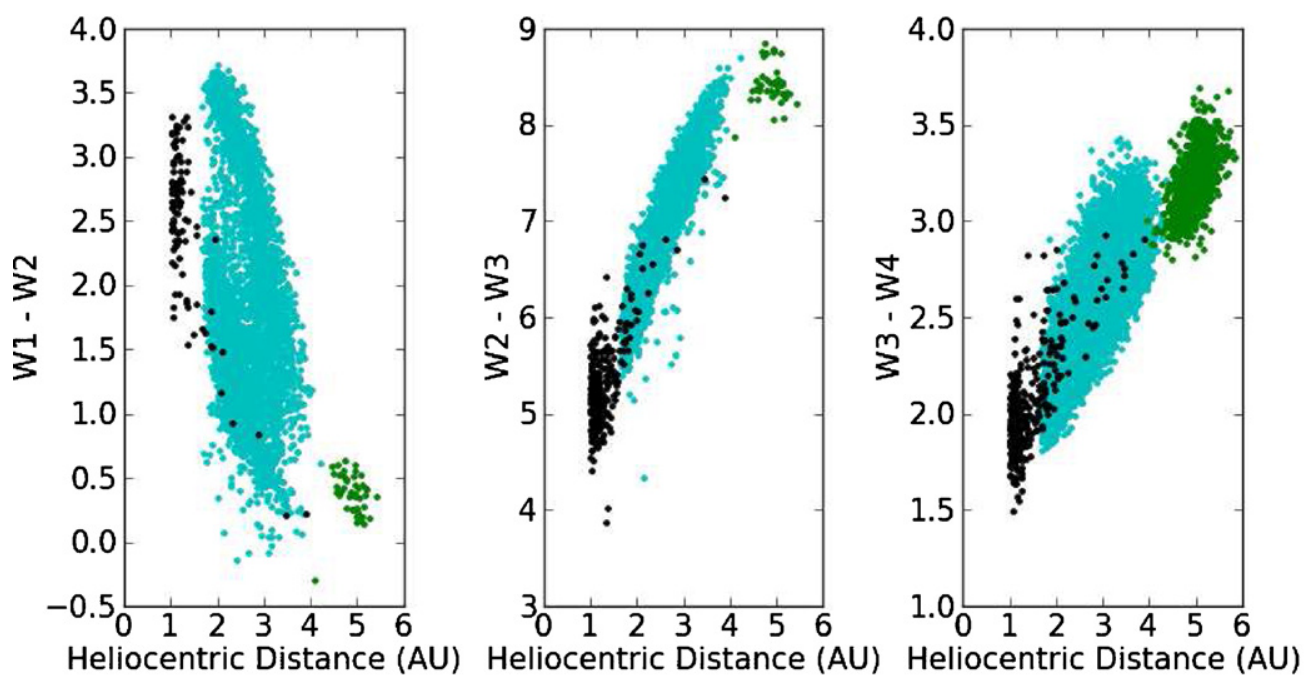

Figure 5. WISE colors vs. heliocentric distance. Black dots are NEOs, cyan dots are Main Belt asteroids, and green dots are Trojan asteroids. (A color version of this figure is available in the online journal.)

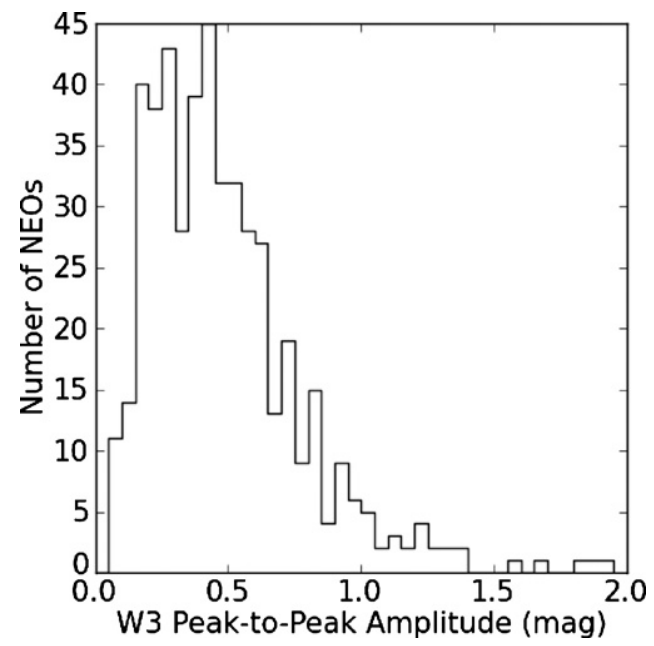

Figure 6. Most NEOs detected by NEOWISE during the cryogenic portion of the mission have $W 3$ peak-to-peak amplitudes equal to $\sim 0.4 \mathrm{mag}$; however, $\sim 18$ NEOs have $W 3$ peak-to-peak amplitudes $>1$. These objects are likely to be highly elongated and possibly binary.

peak-to-peak amplitude in $W 3$ for NEOs detected by NEOWISE during the fully cryogenic portion of the mission. While most objects' amplitudes are $\sim 0.5$ mag or less, there is a fraction of the population with extremely high amplitudes. These objects may be far from spherical, and so the application of a spherical thermal model to these objects may not yield accurate results. These objects are discussed in more detail below.

Thermal models were computed for each WISE measurement, ensuring that the correct Sun-observer-object distances were used. The temperature for each facet was computed, and the Wright et al. (2010) color corrections were applied to each facet. In addition, we adjusted the $W 3$ effective wavelength blueward by $4 \%$ from $11.5608 \mu \mathrm{m}$ to $11.0984 \mu \mathrm{m}$, the $W 4$ effective wavelength redward by $2.5 \%$ from $22.0883 \mu \mathrm{m}$ to $22.6405 \mu \mathrm{m}$, and we included the $-8 \%$ and $+4 \%$ offsets to the $W 3$ and $W 4$ magnitude zero points (respectively) due to the red-blue calibrator discrepancy reported by Wright et al. (2010). The emitted thermal flux for each facet was calculated using NEATM with the bandcenters and zero points given in Wright et al. (2010); the temperature at the anti-subsolar point was set to $3 \mathrm{~K}$, so the facets closest to this point contribute little flux. For NEOs, bands $W 1$ and $W 2$ typically contain a mix of reflected sunlight and thermal emission. The flux from reflected sunlight was computed for each WISE band as described in M11B using the IAU phase curve correction (Bowell et al. 1989). Facets which were illuminated by reflected sunlight and visible to WISE were corrected with the Wright et al. (2010) color corrections appropriate for a G2V star. In order to compute the fraction of the total luminosity due to reflected sunlight, it was necessary to determine the albedo in bands $W 1$ and $W 2, p_{\mathrm{IR}}$. This is discussed in greater detail below.

Physical properties were computed for each object by grouping together observations with no more than a ten day gap between them. This restriction was imposed to ensure that NEOs, which can have significant changes in distance over short times, were modeled accurately.

In general, NEO absolute magnitudes $(H)$ were taken from the MPC's orbital element files, and errors on $H$ were taken to be 0.3 mag. However, updated $H$ magnitudes were taken from the Light curve Database of Warner et al. (2009) for 78 NEOs that were observed by WISE. Emissivity, $\epsilon$, was assumed to be 0.9 for all wavelengths (cf. Harris et al. 2009), and $G$ (the slope parameter of the magnitude-phase relationship) was set to $0.15 \pm$ 0.1 unless a direct measurement from Warner et al. (2009) was available. We note that for some objects with high albedos, the choice of $G=0.15$ may not be appropriate; this is discussed in more detail below.

For objects with measurements in at least two or more WISE bands dominated by thermal emission, the beaming parameter $\eta$ was determined using a least-squares minimization but was constrained to be less than the upper bound set by the FRM case $(\pi)$. Figure 7 shows the fitted $\eta$ values for objects which had measurements in two or more WISE thermal bands, along with a best-fitting double Gaussian distribution. The median value of the 313 NEOs that had fitted $\eta$ was $1.40 \pm 0.5$. The beaming parameter could not be fitted for objects which had observations in only a single WISE thermal band; these objects were assigned $\eta=1.4 \pm 0.5$. Figure 7 also shows fitted beaming as a function of phase $(\alpha)$ along with the best-fitting linear fit, as well as $\eta$ as a function of heliocentric distance and subsolar temperature $\left(T_{\mathrm{ss}}\right)$. The weighted best-fit relationship between $\eta$ and phase $\alpha$ is given by $\eta=(0.00963 \pm 0.00015) \alpha+0.761 \pm 0.009$. 

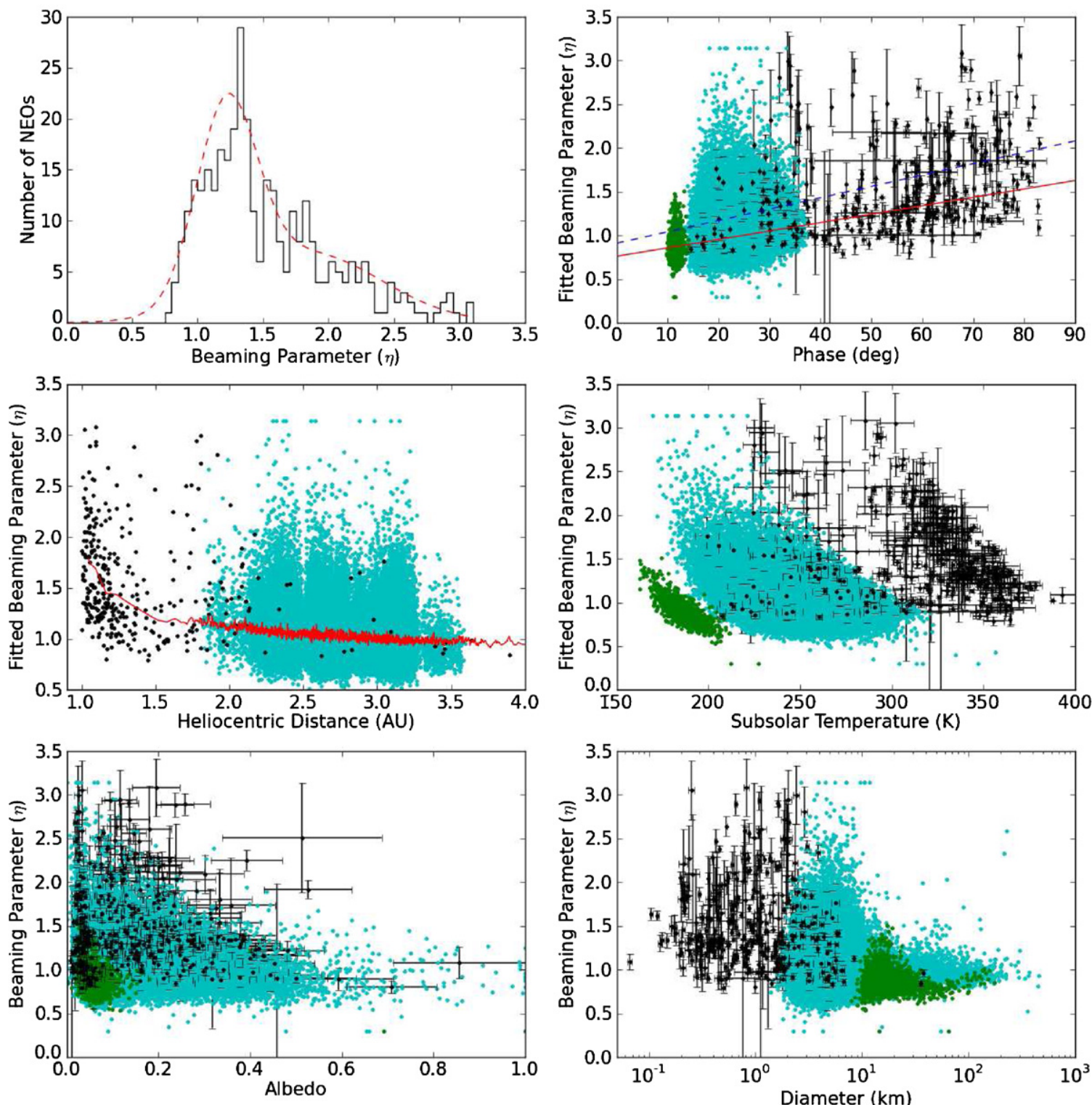

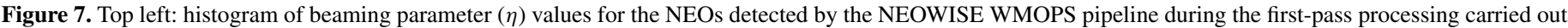

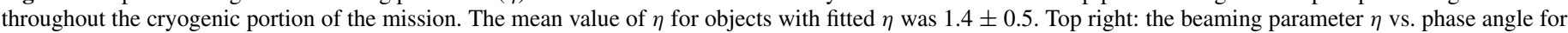

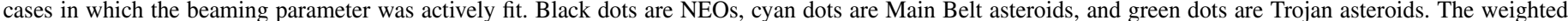

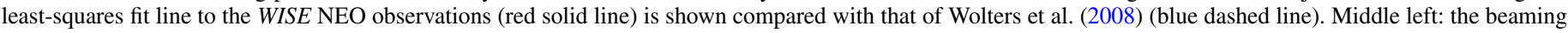

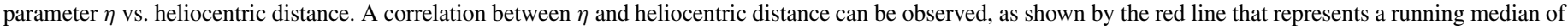
all the objects including NEAs, Mars crossers, and MBAs. Middle right: $\eta$ vs. subsolar temperature. Bottom left: $\eta$ vs. $p_{V}$. Bottom right: $\eta$ vs. diameter.

(A color version of this figure is available in the online journal.)

Figure 7 shows that $\eta$ is strongly correlated with the heliocentric distance at which a given object was observed as shown by the red line representing the running median for the NEOs, Mars crossers, and MBAs; the closer an object is to the Sun, the higher its $\eta$ value for objects observed near $90^{\circ}$ elongation. Because NEATM assumes that an asteroid's night side contributes essentially no thermal flux, objects that are observed at high phase angles (e.g., far away from the subsolar point) may have $\eta$ values that vary in model-dependent ways. Since WISE always observed near $90^{\circ}$ solar elongation, NEOs in particular tended to be observed at high phase angles, meaning that part of the night sides were observed. If the nightside temperature is not zero as assumed by NEATM, the relationship between $\eta$ and heliocentric distance/phase angle can vary depending on the real temperature distribution. While the $\eta$ parameter can serve as an indicator of thermal inertia at low to moderate phase angles, this result suggests that attempting to use $\eta$ as a gauge of thermal inertia or regolith properties at high phase angles must be done with caution, due to the phase-angel dependence of $\eta$. In general, detailed thermophysical modeling should be used if possible to unravel the various thermal and geometric effects contributing to the observed thermal continuum.

As described above, bands $W 1$ and $W 2$ consist of a mix of reflected sunlight and thermal emission for NEOs, and bands $W 3$ and $W 4$ consist purely of thermal emission. In order to properly model the fraction of total emission due to reflected sunlight in each band, it was necessary to determine the infrared albedo $p_{\mathrm{IR}}$. We have made the assumption that $p_{3.6 \mu \mathrm{m}}=p_{4.6 \mu \mathrm{m}}=p_{\mathrm{IR}}$, although it is known that there are a number of material absorption features at these wavelengths (see Gaffey et al. (2002) 

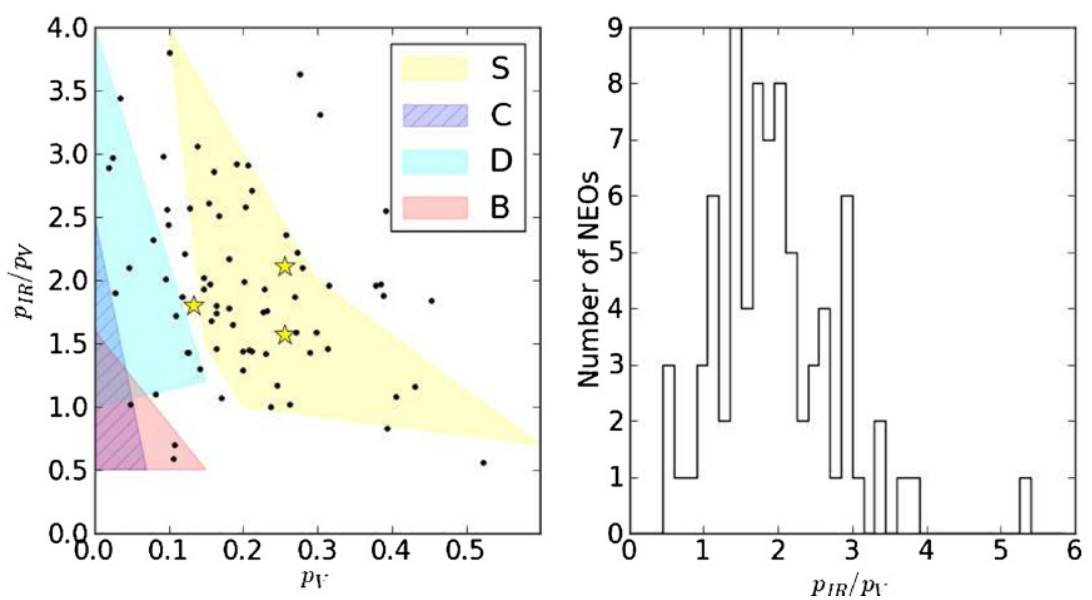

Figure 8. Left: as discussed in Mainzer et al. (2011d), $p_{\mathrm{IR}} / p_{V}$ can be used to distinguish objects with different taxonomic classifications that otherwise have similar $p_{V}$; objects with blue or neutral visible and near-infrared (VNIR) spectral slopes such as C and B types tend to have lower $p_{\text {IR }} / p_{V}$ values than objects with red VNIR slopes, such as $\mathrm{T}$ and $\mathrm{D}$ types. NEAs with low $p_{\mathrm{IR}} / p_{V}$ and low $p_{V}$ are likely to be $\mathrm{C}$ or $\mathrm{B}$ types, and objects with high $p_{\mathrm{IR}} / p_{V}$ but low $p_{V}$ are more likely to be D types. The shaded regions represent the regions covered by various spectroscopically classified Main Belt asteroids shown in Mainzer et al. (2011d). NEOs with known S-type spectroscopic classifications are shown as large yellow stars. Right: histogram of $p_{\mathrm{IR}} / p_{V}$ values for NEOs observed during the cryogenic portion of the mission.

(A color version of this figure is available in the online journal.)

for an overview; the topic is treated in more detail in Mainzer et al. (2011d, hereafter M11D). In order to test the validity of the assumption that the infrared albedo is the same in bands $W 1$ and $W 2$, we recomputed all the thermal model fits with band $W 1$ only and found that the resulting diameters, visible albedos, and infrared albedos were identical to within $\pm 10 \% 1 \sigma$. The geometric albedo $p_{V}$ is defined as the ratio of the brightness of an object observed at zero phase angle to that of a perfectly diffusing Lambertian disk of the same radius located at the same distance. The Bond albedo $(A)$ is related to the visible geometric albedo $p_{V}$ by $A \approx A_{V}=q p_{V}$, where the phase integral $q$ is defined such that $q=2 \int \Phi(\alpha) \sin (\alpha) d \alpha$. $\Phi$ is the phase curve, and $q=1$ for $\Phi=\max (0, \cos (\alpha))$. $G$ is the slope parameter that describes the shape of the phase curve in the $H-G$ model of Bowell et al. (1989) that describes the relationship between an asteroid's brightness and the solar phase angle. For $G=0.15$, $q=0.384$. We make the assumption that $p_{\mathrm{IR}}$ obeys the same relationship, although it is possible it varies with wavelength, so what we denote here as $p_{\text {IR }}$ for convenience may not be exactly analogous to $p_{V}$. We can derive $p_{\text {IR }}$ directly from the WISE objects that have observed reflected sunlight in bands $W 1$ and $W 2$ as well as observations in $W 3$ or $W 4$. Figure 8 shows the ratio of $p_{\mathrm{IR}}$ versus $p_{V}$ for the objects that had fitted $p_{\text {IR }}$. For the NEOs for which $p_{\text {IR }}$ could not be fitted, we used $p_{\mathrm{IR}} / p_{V}=1.6 \pm 1.0$.

As discussed in M11D and Mainzer et al. (2011e, hereafter M11E), asteroids with different taxonomic classes can have different $p_{\mathrm{IR}} / p_{V}$ values despite having similar $p_{V}$; Figure 8 shows the comparison between $p_{V}$ and $p_{\mathrm{IR}} / p_{V}$ for the WMOPSdetected NEOs for which $p_{\mathrm{IR}} / p_{V}$ could be fitted. For example, a correlation was found between spectral classes that have neutral or blue visible/near-infrared (VNIR) spectral types, such as $\mathrm{C}$ or $\mathrm{B}$ types, and objects that have lower values of $p_{\mathrm{IR}} / p_{V}$, even though these spectral classes have nearly identical $p_{V}$. The figure shows the areas where most of the major taxonomic types group, although it should be noted that most of the objects studied in M11D and M11E were MBAs. The extent to which the same groupings in $p_{V}$ and $p_{\mathrm{IR}} / p_{V}$ apply to NEOs is yet to be determined. Taxonomic types with red VNIR slopes such as $\mathrm{T}$ or $\mathrm{D}$ types tend to have higher values of $p_{\mathrm{IR}} / p_{V}$. It is possible that the NEOs shown in Figure 8 with low $p_{V}$ and low $p_{\mathrm{IR}} / p_{V}$ are $\mathrm{C}$ or $\mathrm{B}$ types, whereas those with high $p_{\mathrm{IR}} / p_{V}$ and low $p_{V}$ are more likely to be $\mathrm{T}$ or $\mathrm{D}$ types. As discussed in M11D, the current set of spectroscopically classified objects is heavily affected by selection biases; adding more objects drawn from the NEOWISE sample would improve our understanding of the correspondence between VNIR spectroscopic shapes and features with size, $p_{V}$, and $p_{\mathrm{IR}}$.

\subsection{Thermal Model Error Analysis}

Error bars on $D, p_{V}, p_{\mathrm{IR}}, \eta$, and $T_{\mathrm{ss}}$ were determined for each object by running 50 Monte Carlo trials that varied the objects' $H$ values by the errors described above and the WISE magnitudes by their error bars using Gaussian probability distributions. The minimum magnitude error for all WISE measurements was taken to be 0.03 mag per the in-band repeatability given in Wright et al. (2010), unless the sources were brighter than the limits $W 1=6$, $W 2=6, W 3=4$, and $W 4=0$; these objects were assumed to have magnitude errors of $0.2 \mathrm{mag}$ in their respective bands. The error bar for each object's model magnitude was equal to the weighted standard deviation of all the Monte Carlo trial values. For objects with fixed $\eta$, errors on derived parameters were computed by varying $\eta$ by 0.5 ; this is approximate width of the Gaussian that was fitted to the beaming parameter for objects with fitted beaming (Figure 7). For objects for which $p_{\text {IR }}$ could not be fitted, the Monte Carlo trials varied $p_{\mathrm{IR}} / p_{V}$ by 1.0 .

As described in M11B and Mainzer et al. (2011c), the minimum diameter error that can be achieved using WISE observations is $\sim 10 \%$, and the minimum relative albedo error is $\sim 20 \%$ for objects with more than one WISE thermal band for which $\eta$ can be fitted. Table 1 gives the results of the thermal model fits for the 428 NEOs detected by WMOPS during the fully cryogenic NEOWISE mission using the WISE First Pass data processing pipeline.

\subsection{Interesting NEOs}

\subsubsection{Large Amplitude Brightness Variations}

A number of NEOs stood out as objects of interest. As discussed above, we have identified a number of objects with 

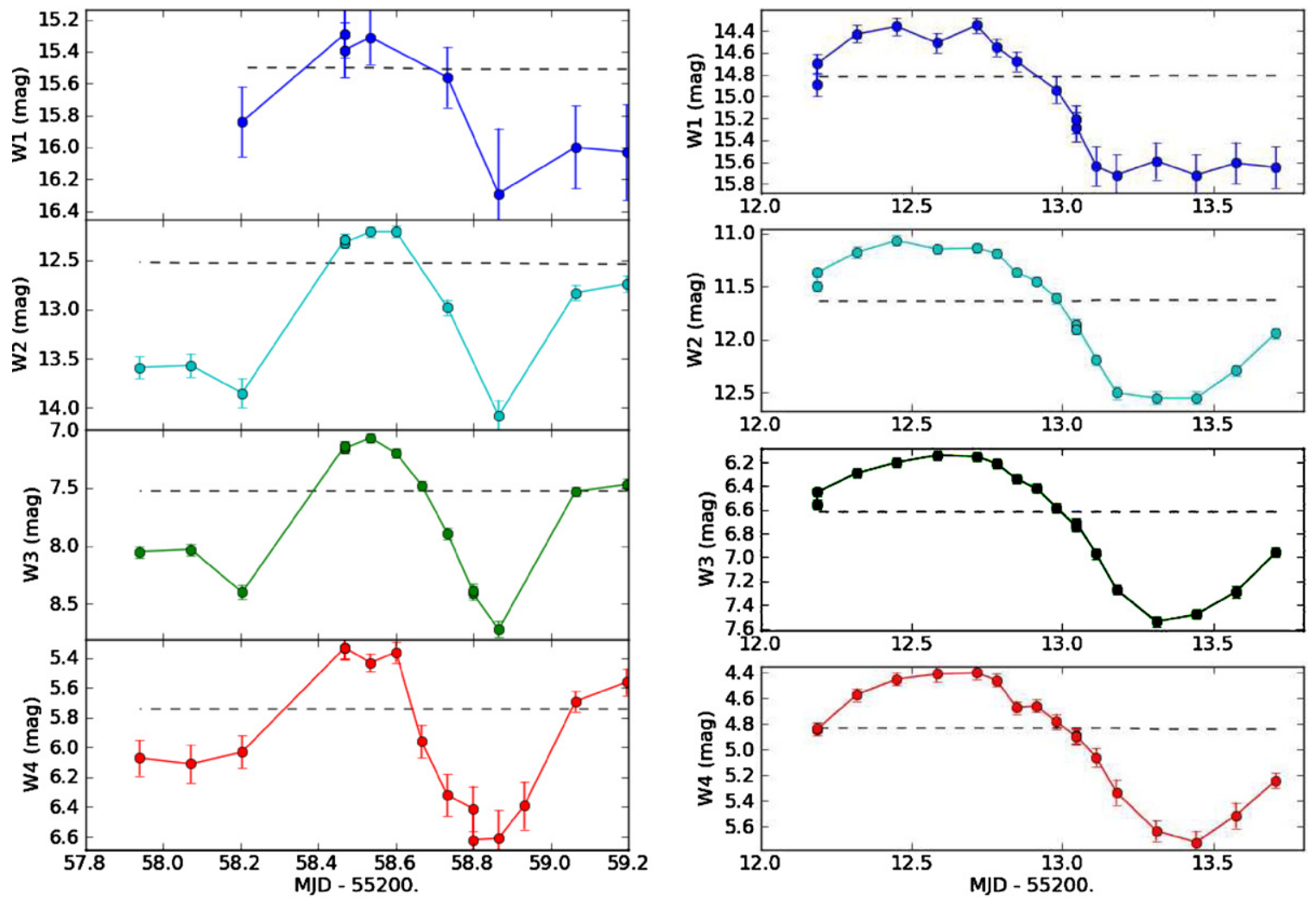

Figure 9. Left: an example of an NEO with a large peak-to-peak light curve variation, (5645). This object has a peak-to-peak amplitude of 1.29 $\pm 0.08,1.65 \pm 0.06$, and $1.87 \pm 0.08 \mathrm{mag}$ in bands $W 2, W 3$, and $W 4$, respectively. (5645) is known to have a $30.39 \mathrm{hr}$ rotational period measured from visible photometry and is likely to be tumbling (Warner et al. 2009). A future work will convolve the visible light curves of this and other objects with the NEOWISE infrared detections. Right: NEO 2009 WO6 also has a large-amplitude magnitude variation that is observed in all four WISE wavelengths.

(A color version of this figure is available in the online journal.)

large-amplitude peak-to-peak variations. Figure 9 shows examples of NEOs with very large amplitude magnitude variations, indicating that these objects are likely to be highly elongated. WISE preferentially observed NEOs at higher phase angles, which may skew the observed amplitude. Effective diameters derived from the application of a spherical NEATM model to such objects must be viewed with caution. These objects would benefit both from obtaining additional observations in visible light to better constrain their shapes and rotational states as well as modeling them as non-spherical shapes.

Many NEOs in the NEOWISE data set are observed to have larger peak-to-peak amplitude variations in bands $W 1$ and $W 2$ as compared with $W 3$ and $W 4$. This is possibly due to the fact that thermal emission can emanate from beyond the terminator on an asteroid, depending on its thermal inertia, whereas reflected sunlight tends to come from only those portions that are illuminated. Since bands $W 1$ and $W 2$ consist of a mix of reflected sunlight and thermal emission, and since some NEOs have higher thermal inertias than others, a discrepancy between amplitudes at the different wavelengths can occur. An example of such an object can be found in NEO (1865) Cerberus (Figure 10). In addition to having significantly higher peakto-peak light curve amplitudes in bands $W 1$ and $W 2$ than in bands $W 3$ and $W 4$, this object has a high beaming parameter, $\eta=2.94 \pm 0.03$. This is close to the maximum $\eta$ of $\pi$ allowed by the FRM, which postulates that the object's cooling time is slow compared to its rotational rate; however, as shown in Figure 7 and as discussed above, $\eta$ is correlated with heliocentric distance for WISE-observed NEOs, and (1865) was located
1.09 AU from the Sun when it was detected by NEOWISE. The object has a known rotational period of $6.810 \mathrm{hr}$ (Warner et al. 2009), and this appears consistent with the rotational rate that can be observed in the figure. It may be that for this object, the assumption that temperature decreases as

$$
T(\theta, \phi)=T_{\mathrm{ss}}[\max (0, \cos \theta \cos \alpha+\sin \theta \sin \alpha \cos \phi)]^{1 / 4}
$$

is inappropriate, and an improved model of temperature distribution may be desirable. NEATM assumes that $T(\theta, \phi)$ goes to zero on the night side of the asteroid, even though this may not be correct. A number of other NEOs in our sample have high beaming parameters, and these objects are candidates for having high thermal inertia and/or rapid rotational rates. Full exploration of the true physical meaning of the relationship between $\eta$, heliocentric distance, rotational rate, and properties such as thermal inertia will be the subject of future work.

\subsubsection{Multi-epoch Observations}

Due to the WISE observing cadence, there were 55 NEOs in our current sample during the fully cryogenic portion of the mission that were observed at two separate epochs; three NEOs were observed at three epochs. Of these, 20 have diameter measurements that agree to within $10 \%$ between all available epochs; the remaining 35 objects' diameters agree to better than $10 \%$ between epochs. However, three NEOs have diameter measurements that are disparate at the $30 \%$ or greater level. Of these, all have $W 2$ or $W 3$ peak-to-peak amplitudes larger than $0.3 \mathrm{mag}$, indicating that they are likely to have elongated shapes. Elongated objects observed at different viewing angles are likely 


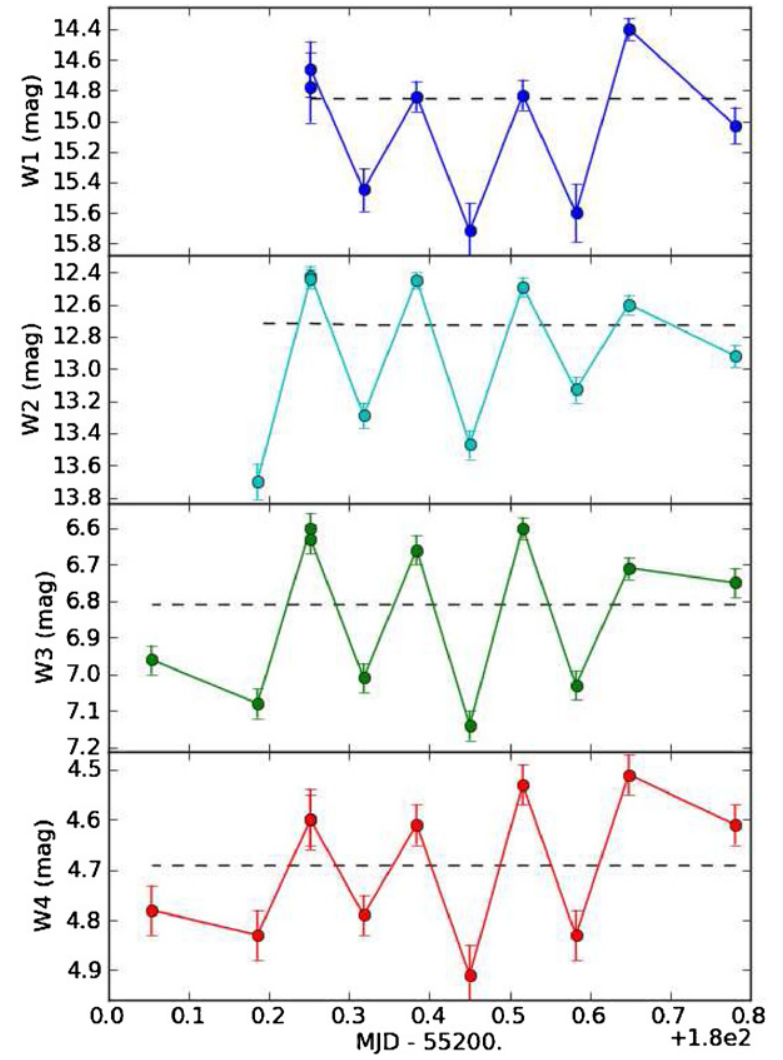

Figure 10. NEO (1865) Cerberus has a significantly larger light curve amplitude at the shorter WISE wavelengths than the longer ones: $1.31 \pm 0.12,1.28 \pm 0.08$ $0.54 \pm 0.06$, and $0.39 \pm 0.07$ in bands $W 1, W 2, W 3$, and $W 4$ respectively. Cerberus is known to have a $6.810 \mathrm{hr}$ rotational period (Warner et al. 2009), and its WISE-derived diameter is $1.6 \mathrm{~km}$. The high beaming parameter $\eta=2.94 \pm 0.03$ suggests that this could be an object with high thermal inertia; however, objects with low heliocentric distances tend to have higher $\eta$ values. The difference in amplitudes between shorter and longer WISE wavelengths could be due to the fact that thermal emission, which dominates bands $W 3$ and $W 4$, can come from a larger fraction of the object's total visible area than can reflected sunlight, particularly if it has high thermal inertia and therefore a more uniform temperature distribution.

(A color version of this figure is available in the online journal.)

to be poorly fit by a spherical thermal model. Furthermore, large disparities between observing epochs can be indicative of large differences between temperature on the evening and morning sides; more sophisticated thermal models will need to be developed. In order to compute a single effective diameter and albedo for purposes of computing the debiased population statistics, the thermal model was fit using observations from all epochs simultaneously. These single effective diameters were used as an input to the debiasing model; nevertheless, these objects should be fit with non-spherical thermal models. The objects with relatively low amplitude light curve variations that have large discrepancies in their fitted diameters and albedos between the two epochs are possibly good candidates for having significant temperature differences between their morning and evening sides. Objects with large morning/evening temperature differences could be subject to increased Yarkovsky forces.

\section{DEBIASING}

In order to determine the size and albedo distributions of the NEOs, it is necessary to remove the effects of any systematic survey biases present in the NEOWISE sample. NEOWISE carried out a "blind" search for moving objects, meaning that all moving objects were detected in the same way regardless of whether or not they had been previously discovered by another observer. This means that the NEOWISE survey can be debiased independently of the biases of other surveys with the following caveats: there were 12 objects that were designated without visible follow-up and 22 objects that appeared on the NEO Confirmation Page but received neither designations nor followup; there is an undetermined number of tracklets that may have been misclassified by the MPC as non-NEOs. Future work will attempt to assess the fraction of real NEOs that are misclassified.

In order to model the NEOWISE survey biases, a high-fidelity simulation of the NEOWISE survey was created. As described in M11A and the WISE Explanatory Supplement, the WMOPS pipeline required five independent detections of a particular object for it to have been considered detected. Sources were extracted down to $4.5 \sigma$ in the Level $1 \mathrm{~b}$ images. We used the Julian dates and coordinates of the centers of all pointings that were used by WMOPS throughout the fully cryogenic portion of the survey as well as the on-sky footprint of each pointing $(47 \times$ $47 \mathrm{arcmin})$ to recreate the survey history. As described in Wright et al. (2010), the WISE survey includes hundreds of observations of the ecliptic poles and an average of 10-12 observations of most moving objects in the ecliptic plane. Furthermore, due to the observational cadence described in M11A and Wright et al. (2010), WMOPS is sensitive to objects with an apparent velocity between 0.06 and 3.2 day $^{-1}$. The simulated survey replicated these selection criteria for identifying a moving object tracklet.

We first determined the number of NEAs with effective diameters larger than $1 \mathrm{~km}$ by collecting together as much information as possible about all known NEAs (whether observed by WISE or by others) with $H<22$, since that is the approximate limit at which an asteroid with $p_{V}=1 \%$ could still have $D=1 \mathrm{~km}$. Where possible, we used diameters determined from radar observations, in situ spacecraft imaging, or radiometric measurements from WISE, IRAS, or ground-based thermal infrared observations. With NEOWISE, we detected 175 NEOs with $D>1 \mathrm{~km}$; when we added in all the NEOs with measured diameters that we could find from the literature, we found an additional 75 objects, for a total of 250 objects with previously known effective diameters that are $>1 \mathrm{~km}$. We also found from the literature an additional 72 NEAs with taxonomic classifications and assigned them approximate albedos based on the average albedos given in Table 1 of M11D. We note that most of the objects studied in M11D were MBAs, and albedos associated with a particular taxonomic class were found to be strongly affected by selection biases below $\sim 30 \mathrm{~km}$. The extent to which the albedo distributions for various taxonomic classes for the NEOs resemble those of large MBAs is unknown, since the spectroscopic samples used in M11D and M11E are heavily biased against optically faint objects, which preferentially tend to be small, low albedo objects. Using the albedo distributions for different taxonomic classes derived from large MBAs introduces an unknown error, and the error estimates we quote here must be regarded as lower limits. Our estimates will be improved by finding more NEOs within the WISE data set and directly computing diameters for them. We used the albedo distribution of the 250 objects with known diameters to compute $D$ for the previously known NEOs with $H<22$ mag but no diameter or albedo measurements using the relationship

$$
p_{v}=\left[\frac{1329 \times 10^{-0.2 H}}{D}\right]^{2} \text {. }
$$



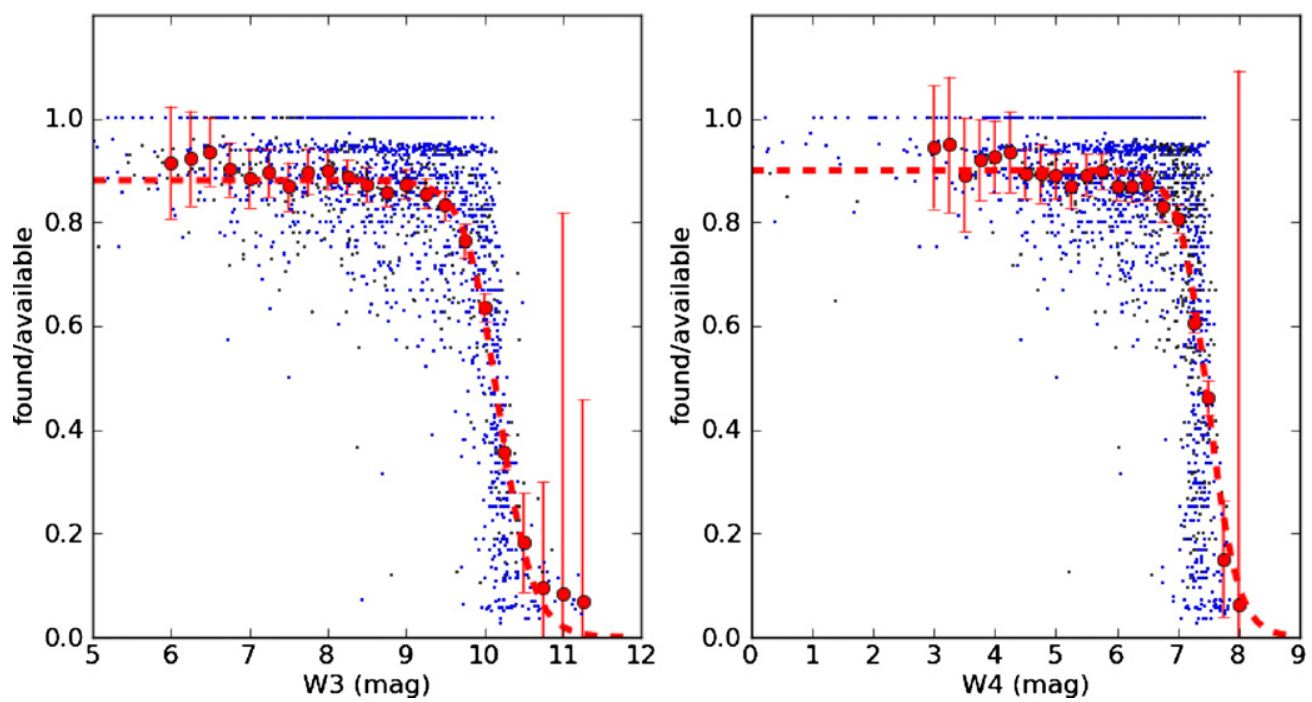

Figure 11. Known Solar System Object Possible Association List was used to assess the solar system object detection probability of the NEOWISE survey during the cryogenic portion of the mission across the sky. This figure shows an example of the detection probability for a $40^{\circ} \times 20^{\circ}$ region centered at $0^{\circ}$ ecliptic latitude and $0^{\circ}$ ecliptic longitude. The dots show $n_{\text {detected }} / n_{\text {available }}$ as a function of observed magnitude for individual numbered asteroids, where $n_{\text {detected }}$ is the number of times

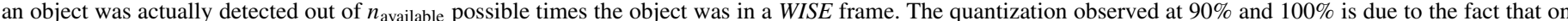
average, WISE observes most moving objects $\sim 10$ times, and bright objects are usually detected 9 or 10 times. The red circles are the medians of $n_{\text {detected }} / n_{\text {available }}$ for each magnitude bin. The function given in Equation (2) was fitted to the red circles (dashed red line); this represents the detection probability.

(A color version of this figure is available in the online journal.)

Applying the albedo distribution of the $H<22$ objects with known diameters gave us a total of $911 \pm 17$ previously known NEOs with diameters $>1 \mathrm{~km}$.

Next, it was necessary to determine the remaining population of $1 \mathrm{~km}$ NEOs likely to exist that have not yet been discovered. NEOWISE discovered 16 new NEOs with $D>1 \mathrm{~km}$. The total undiscovered population remaining was found by determining the NEOWISE survey bias for objects in this size range, then debiasing the NEOWISE discoveries of large NEOs. The survey bias was found by creating a synthetic population of large NEOs and running a simulated WMOPS survey on this population to identify what would have been found. In order to produce a statistically valid sample, we created 25 populations each with 50,000 synthetic NEOs with orbital elements generated randomly using the synthetic solar system model (S3M; Grav et al. 2011). In the S3M model, the NEO orbital elements are generated according to the distribution created from five source regions given in Bottke et al. (2002). Visible albedo and $p_{\mathrm{IR}}$ were randomly assigned values between zero and one; $\eta$ was randomly assigned a value between zero and $\pi$. Diameter was assigned a random value between 1 and $10 \mathrm{~km}$. The next step was to compute the survey bias by integrating the synthetic objects' orbits over the entire list of $\sim 700,000$ pointings carried out during the cryogenic portion of the WISE survey and see which objects were present in each frame. We then determined whether or not the objects would have been detected by NEOWISE by modeling their fluxes and comparing them to a model of the WISE sensitivity to moving objects in all four bands. Several other trial diameter, $\eta$, and $p_{V}$ distributions were used, and as expected, the survey bias is not sensitive to the input distributions used as long as there are enough synthetic objects in each bin to reduce statistical error from the simulation.

We used the WISE Known Solar System Object Possible Association List (KSSOPAL) to assess the survey detection efficiency to moving objects at various places across the sky. KSSOPAL uses a list of known minor planet ephemerides to predict where asteroids should be in each WISE frame and to generate a list of probable matches; however, unlike WMOPS, it makes no attempt to eliminate matches to inertially fixed sources such as stars or galaxies, nor does it remove spurious associations with artifacts or cosmic rays. We searched the KSSOPAL for numbered asteroids only as these generally have well-determined orbits. In order to reduce the possibility of spurious associations with stars and galaxies, we checked each source location from KSSOPAL against the WISE Level 3 Atlas source table and used the $n$ out of $m$ statistics provided to search for sources that repeated; these sources were flagged. For each magnitude bin, we computed the total number of available detections predicted by KSSOPAL and compared this to the total number of matches found. This result, shown in Figure 11, gives an estimate of the single image completeness as a function of flux for a particular region of the sky for bands $W 3$ and $W 4$. We computed this completeness curve for a number of different locations throughout the sky to bracket the WISE survey sensitivity as a function of ecliptic latitude/longitude and distance from the Galactic center. Figure 11 shows the probability that a moving object of a particular flux was detected by the WISE pipeline at a particular location on the sky. Based on the results from KSSOPAL, we found that the detection probability dropped to zero within $5^{\circ}$ of the Galactic center. The detection probability curves $P$ were fitted with the following function for both bands $W 3$ and $W 4$ :

$$
P=\frac{a_{0}}{2}\left[1-\tanh \left(a_{2}\left(W-a_{1}\right)\right)\right],
$$

where $W$ is the $W 3$ or $W 4$ magnitude and $a_{i}$ are the fitted coefficients. For sources between $5^{\circ}$ and $25^{\circ}$ of the Galactic center, the detection probability coefficients were given by $a_{0}=0.9, a_{1}=9.5, a_{2}=1.0$ for band $W 3$ and $a_{0}=0.9$, $a_{1}=7.0, a_{2}=2.0$ for band $W 4$; for sources at all further distances from the Galactic center, the coefficients were given by $a_{0}=0.9, a_{1}=10.25, a_{2}=2.5$ for band $W 3$ and $a_{0}=0.93$, $a_{1}=7.5, a_{2}=2.1$ for band $W 4$.

The synthetic population of NEAs with $D>1 \mathrm{~km}$ was compared to the list of frames taken throughout the cryogenic 

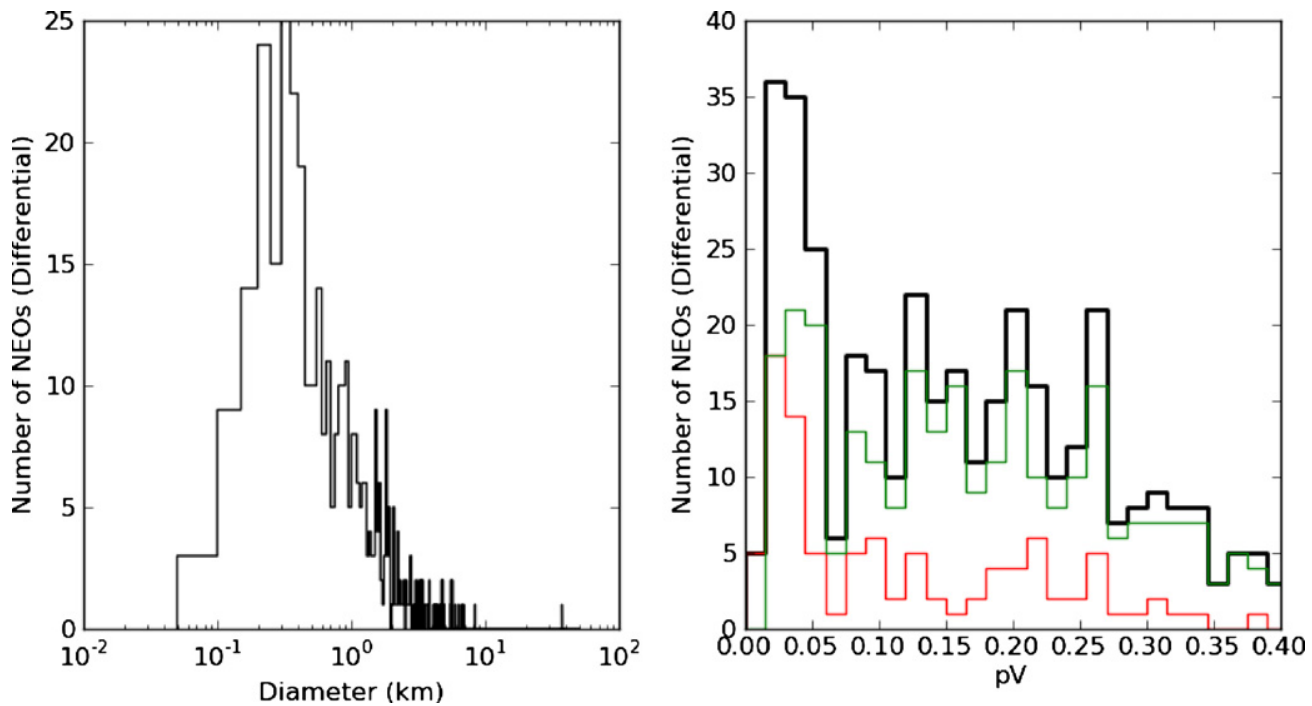

Figure 12. Left: preliminary observed undebiased differential diameter distribution for the NEOs observed during the cryogenic portion of the mission. The peak of the distribution occurs at $\sim 300 \mathrm{~m}$. Right: the albedo distribution for all NEOs observed by NEOWISE during the cryogenic portion of the mission are shown as the heavy black line; previously known NEOs are shown in green, and NEOs discovered by NEOWISE are shown in red. There are proportionately more low-albedo NEOs that were discovered by NEOWISE than in the previously known population; this is evidence of the bias that visible surveys suffer against low-albedo objects. (A color version of this figure is available in the online journal.)

portion of the survey to determine whether or not a particular object was present in the WISE field of view. Known comets were not considered in the population at this point. As discussed above, physical parameters were randomly assigned to each object and fluxes were computed using the NEATM model (the observed size and albedo distributions are shown in Figure 12). In order for an object to be detected by the synthetic survey, it had to meet the WMOPS selection criteria: the object had to appear five or more times and had to have a relative velocity in the range $0.06-3.2$ day $^{-1}$. We note that the WMOPS software is not sensitive to the fastest-moving NEOs, which will tend to be small objects that are very close to Earth. This bias against fast-moving NEOs will result in an additional loss of sensitivity to the smallest objects; we will attempt to model this bias in future work. The survey bias for objects with $D>1 \mathrm{~km}$ was found by dividing the population of synthetic objects in each size bin by the population found by the simulated NEOWISE survey. The survey bias in diameter for objects $>1 \mathrm{~km}$ is shown in Figure 13 along with the NEOWISE-discovered NEAs. The total number of remaining undiscovered NEAs $>1 \mathrm{~km}$ was computed by dividing the NEOWISE-discovered population's differential size distribution by the survey bias, then integrating the result over diameter, resulting in $\sim 80 \pm 9$ NEAs remaining to be discovered in this size range. When added to the $911 \pm 17$ previously known NEAs, we compute a total of $981 \pm 19$ NEAs with $D>1 \mathrm{~km}$. This result suggests that the Spaceguard goal of detecting $90 \%$ of all NEOs larger than $1 \mathrm{~km}$ (Morrison 1992) has essentially been met (although we note that our analysis does not yet address the NECs).

We do not find strong evidence for a correlation between diameter and $p_{V}$ from the NEOWISE data set. Harris (2006) and Delbó et al. (2003) show an apparent correlation between size, $p_{V}$, and asteroid taxonomic type. In M11D, we show that $p_{V}$ is correlated with diameter within a particular taxonomic type, but this correlation could be partially or entirely due to the observational biases of visible light surveys and spectroscopic measurements, which are less likely to detect and characterize small asteroids with lower albedos. Figure 14 shows a plot of diameters versus $p_{V}$ for all the NEOs observed during

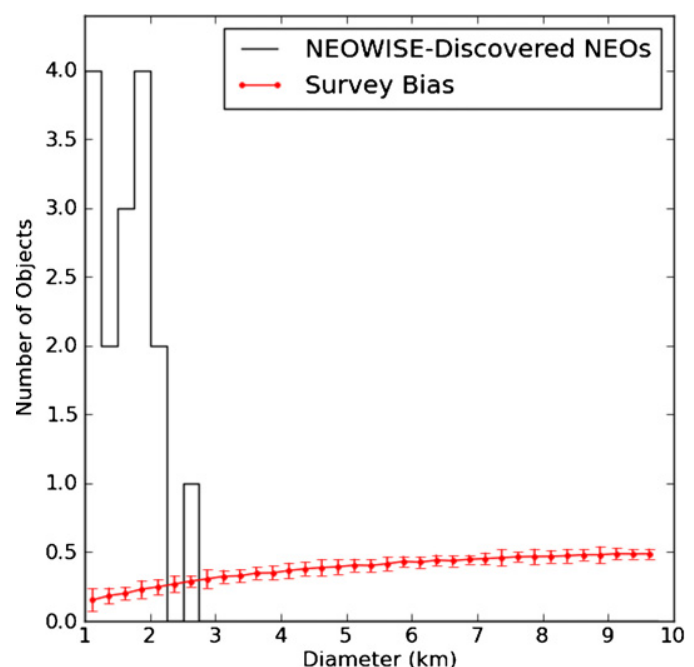

Figure 13. NEOWISE survey bias for objects with $D>1 \mathrm{~km}$ (red line with points) was computed by creating a synthetic population of NEOs with orbits generated using the orbital element distribution of Bottke et al. (2002). The number of NEOWISE-discovered NEOs $>1 \mathrm{~km}$ is shown as the solid black line. The total number of remaining undiscovered NEOs was determined by dividing the NEOWISE-discovered NEO distribution by the survey bias, then integrating the result over diameter.

(A color version of this figure is available in the online journal.)

the cryogenic portion of the NEOWISE survey. No strong correlation can be observed. Furthermore, it should be noted that there were 12 NEOs that received designations from the MPC but did not have visible light follow-up, so $p_{V}$ could not be computed for these objects. They tended to be the most difficult for visible light observers to follow-up, implying that they are likely to have low albedos. An additional 22 candidate NEOs appeared on the NEOCP but were not designated due to their short observational arcs, so neither diameters nor $p_{V}$ could be computed for these objects. Like the 12 that were designated, these objects were the most difficult for observers to detect (however, it is possible that some of these undesignated objects are MBAs or Trojans instead of NEOs). Finally, as discussed 


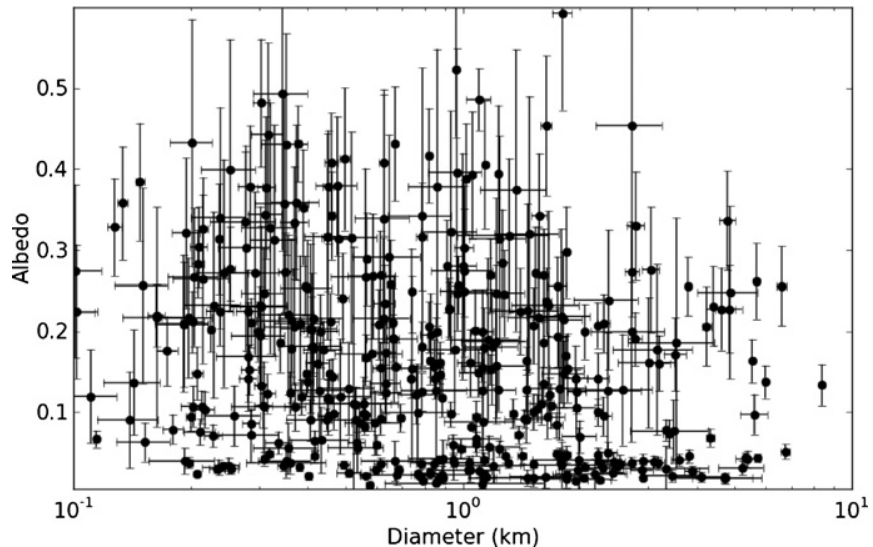

Figure 14. We do not observe a strong correlation of $p_{V}$ with diameter for the objects detected during the cryogenic portion of the NEOWISE survey. Missing from the plot are the 12 NEOs that received designations but received no visible light follow-up, along with 22 candidate NEOs that appeared on the NEO Confirmation Page but received neither designations nor visible follow-up. These objects are likely to be both small and dark. above, WMOPS is less sensitive to the fastest moving NEOs, which are likely to be small objects very close to the Earth, resulting in a further bias against the smallest objects. These fastmoving small NEOs are also likely to be subject to additional bias if they have low albedos, since we require visible light follow-up to obtain an albedo measurement. The 12 designated NEOs for which $p_{V}$ could not be determined represent only $\sim 5 \%$ of the 460 objects detected during the cryogenic portion of the survey, so they can only have a commensurately small effect on the error in the population statistics. A more detailed treatment of these objects, the 22 without designations, and the bias against fast-moving NEOs will be dealt with in a future work. For now, the error estimates presented herein must be regarded as a lower limit, and with these caveats, we conclude that there is no strong dependence of $p_{V}$ on diameter. The $p_{V}$ distribution shown in Figure 12 was assumed to hold down to the smallest sizes. While a rigorous comparison of our results with those of earlier studies (e.g., Delbó et al. 2003; Harris 2006) would require data for NEOs of the same taxonomic types in the same size range, our result suggests that the apparent correlations between size and albedo reported by others could be entirely due to observational biases and small sample sizes as was also shown in Mainzer et al. (2011d). Linkages between albedo, size and
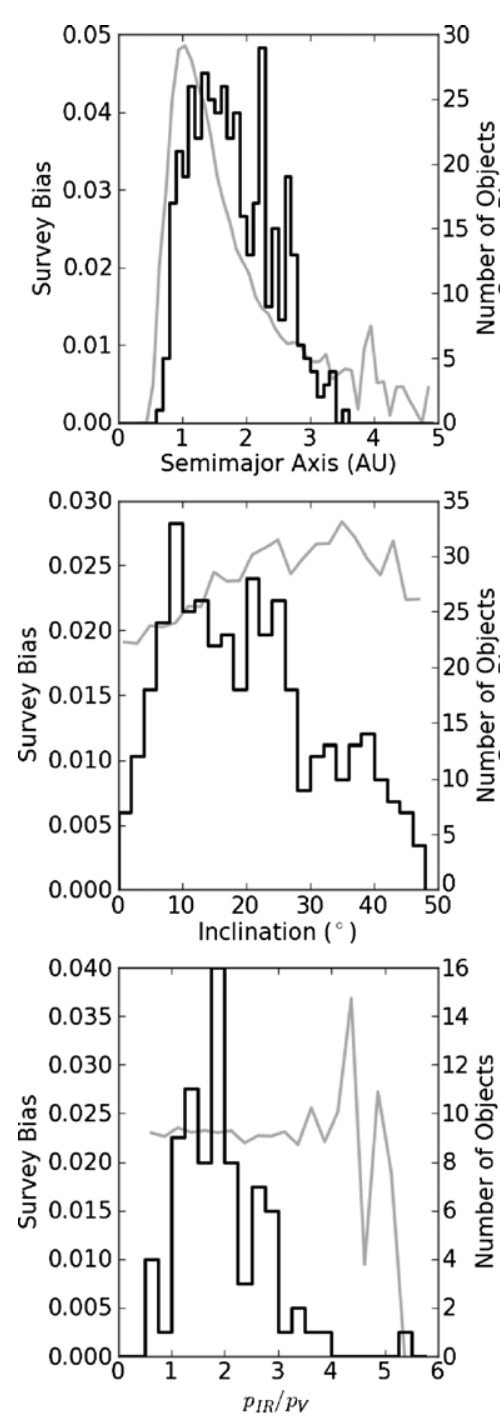
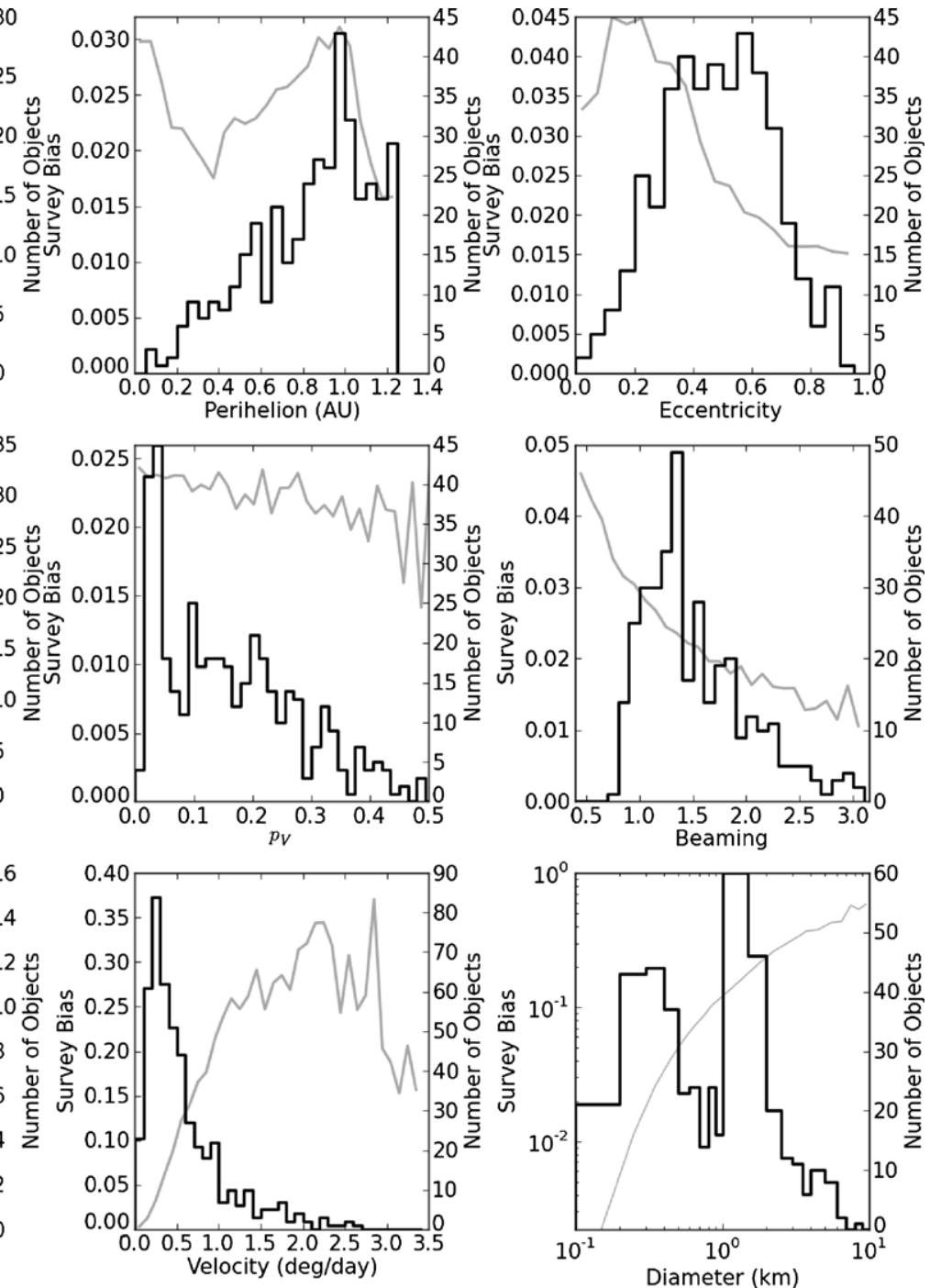

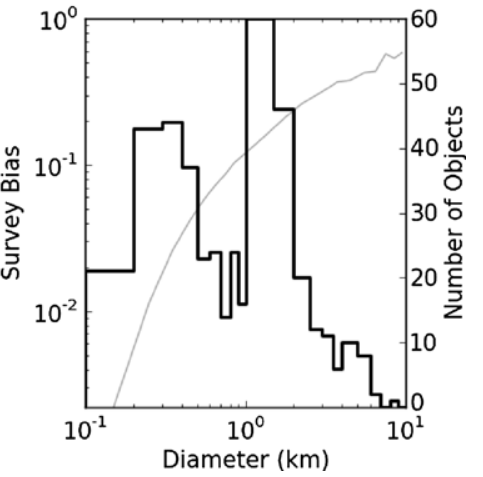

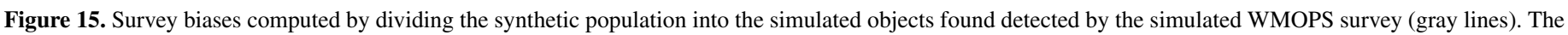
black lines show the properties of the objects detected by WMOPS. It can be seen that the WISE survey is essentially unbiased with respect to visible albedo. 

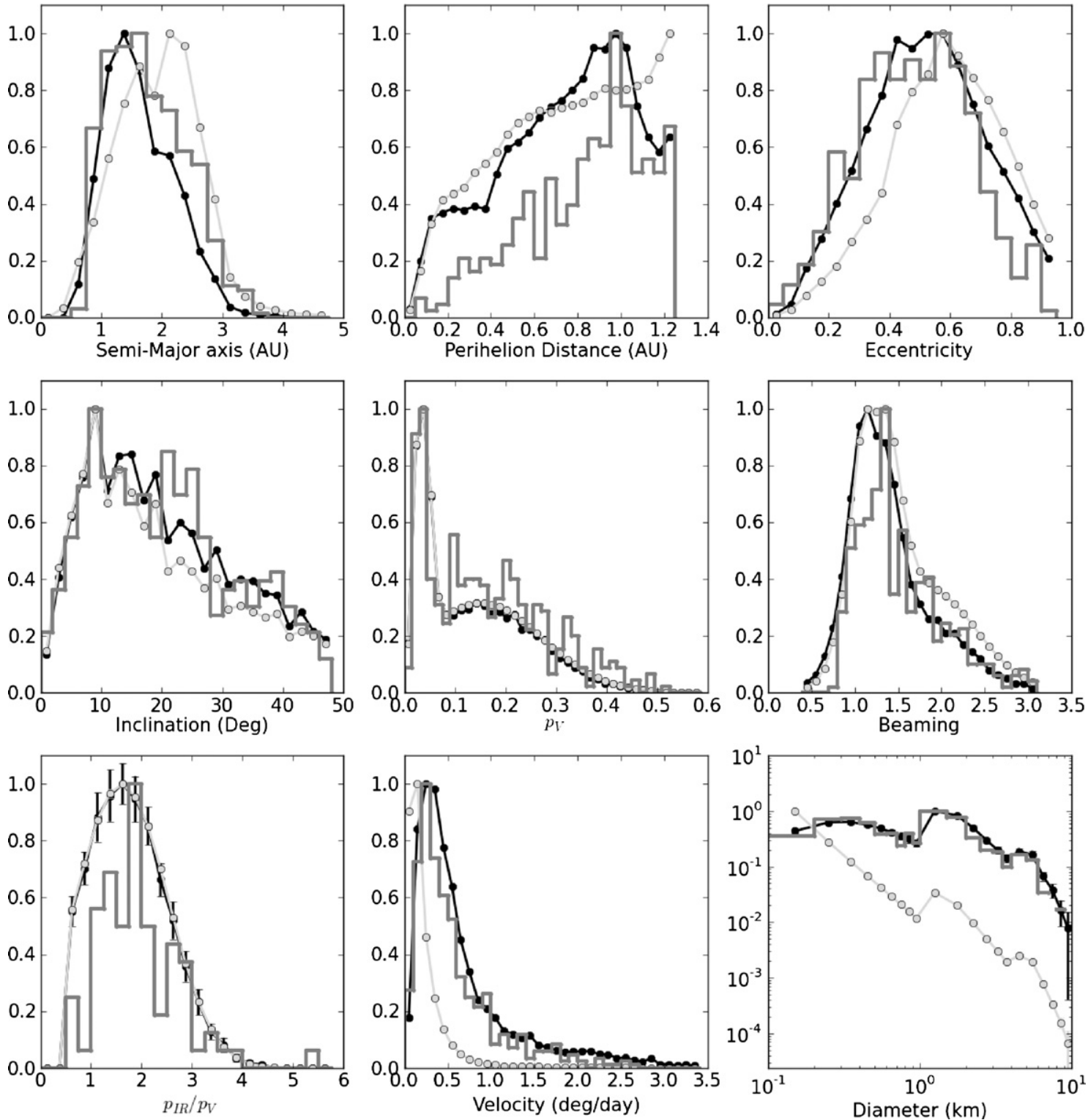

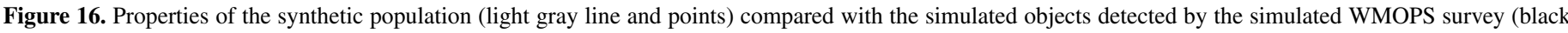

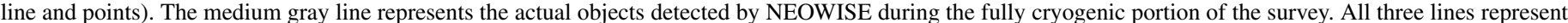

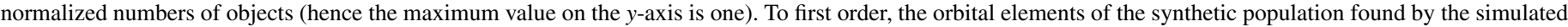

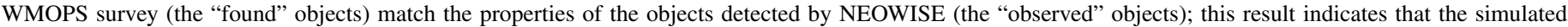

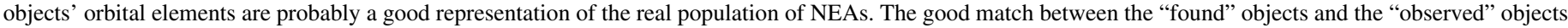

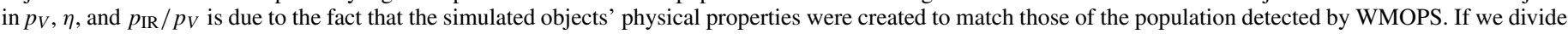

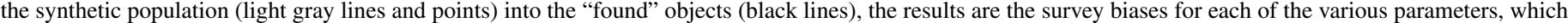
are shown as the light gray lines in Figure 15.

space weathering cannot be reliably made without accounting for these biases.

With the total number of $1 \mathrm{~km}$ NEAs in hand as well as the observed distributions for $p_{V}, p_{\mathrm{IR}} / p_{V}$, and $\eta$, it was possible to compute the total numbers of objects at smaller size ranges. As before, the total number of objects with diameters $>100 \mathrm{~m}$ was determined by computing the survey diameter bias in each size bin and dividing it into the NEOWISE-observed diameter distribution. As was done for the $>1 \mathrm{~km}$ NEAs, the survey biases down to $100 \mathrm{~m}$ were determined by creating a synthetic population of objects and computing which synthetic objects would have been detected by WMOPS. Instead of creating synthetic objects with flat distributions for $p_{V}, p_{\mathrm{IR}} / p_{V}$, and $\eta$, we used the distributions for the objects that were observed by the NEOWISE survey (Figures 12, 8, and 7). The visible albedo was assigned using a probability function described by a double Gaussian:

$$
P\left(p_{V}\right)=v_{0} e^{-\left(p_{V}-v_{1}\right) / 2 v_{2}^{2}}+v_{3} e^{-\left(p_{V}-v_{4}\right) / 2 v_{5}^{2}},
$$

where $v_{0}=12.63, v_{1}=0.034, v_{2}=0.014, v_{3}=3.99$, $v_{4}=0.151$, and $v_{5}=0.122$. The beaming parameter $\eta$ was chosen using a double Gaussian probability distribution, and $p_{\mathrm{IR}} / p_{V}$ was generated using a single Gaussian probability distribution.

The cumulative diameter distribution was initially modeled as a broken power law $\left(N>D^{-\alpha}\right)$ with $\alpha=5$ above $5 \mathrm{~km}$ and $\alpha=2.1$ for $D<5 \mathrm{~km}$ based on Jedicke et al. (2002). Fluxes were generated for each synthetic object using the assigned physical parameters and orbits as inputs to the faceted NEATM model described above. The broken power law was found to be 


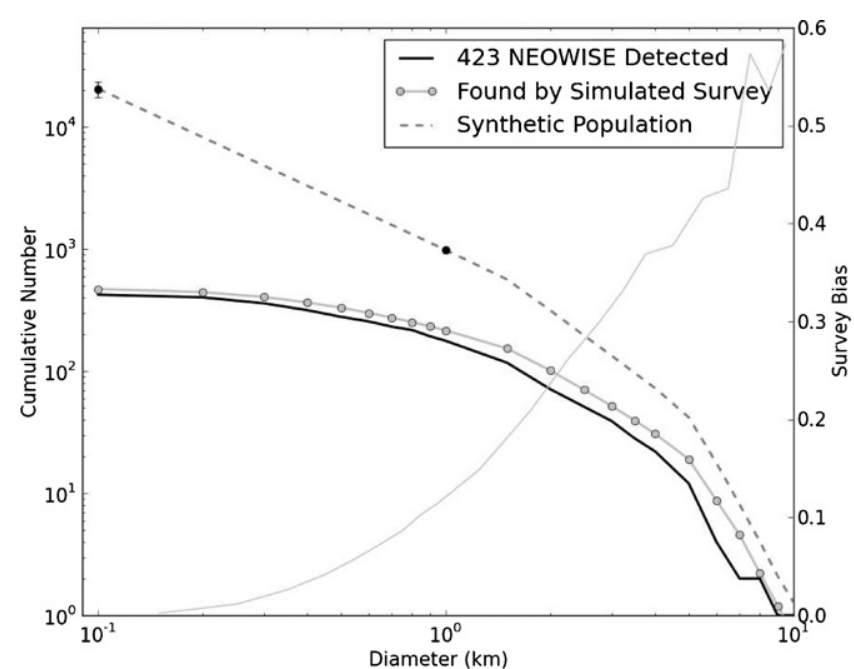

Figure 17. Cumulative size distribution of the synthetic population, the synthetic objects found by the simulated WMOPS survey, and the objects detected by NEOWISE. This synthetic population of objects (gray dots) has a best-fit slope of $1.32 \pm 0.14$ below $1.5 \mathrm{~km}$. The black dots represent typical error bars on the total number of objects at each size range.

a poor match to the observed cumulative diameter distribution, producing too many NEAs at the smallest sizes. The cumulative size distribution was found to be best represented by a triple power law with breaks at $5 \mathrm{~km}$ and $1.5 \mathrm{~km}$. With this functional form of the cumulative size distribution, we found the slope of the power law below $1.5 \mathrm{~km}$ by computing the total number of NEAs with diameters $>100 \mathrm{~m}$ as described above by dividing the observed differential size distribution by the survey bias, then integrating the result over diameter. Using this method, we computed a total of $20,500 \pm 3000$ NEAs with $D>100 \mathrm{~m}$. Using this number and the number of $1 \mathrm{~km}$ NEAs results in a slope of the cumulative size distribution power law of $1.32 \pm$ 0.14 (Figure 17). This power-law slope produces $\sim 13,200 \pm$ 1900 NEAs with $D>140 \mathrm{~m}$, the limit of the George E. Brown Congressional mandate, and $\sim 51,300 \pm 11,500$ NEAs with $D>50 \mathrm{~m}$. This result is lower than was predicted by previous analyses such as Harris (2008), Jedicke et al. (2002), and Rabinowitz et al. (2000). The break in the power law at $1.5 \mathrm{~km}$ is similar to that shown in Harris (2008). Harris (2008) shows another break in the cumulative size distribution at $D \sim 50-100 \mathrm{~m}$ and finds that the slope of the size distribution steepens, indicating that there are larger numbers of smaller objects in this size range. However, as our current sample only includes four NEAs with $D<100 \mathrm{~m}$, our determination of the numbers of objects or the slope of the size distribution below $50-100 \mathrm{~m}$ is not reliable.

The survey biases in $p_{V}, p_{\mathrm{IR}} / p_{V}, \eta$, semimajor axis, eccentricity, inclination, and perihelion were determined by dividing the total synthetic population into the distributions of the objects that would have been found by the NEOWISE survey (Figure 15). The results show that the NEOWISE survey is essentially unbiased with respect to $p_{V}$; this is expected given the weak dependence of thermal flux on $p_{V}$. Because the WISE survey covered the entire sky, including the ecliptic poles, the survey has a moderate bias in favor of high inclination objects. Comparison of the synthetic objects' orbital elements produced by the Bottke et al. (2002) model to the observed orbital element distributions reveals that this model is reasonably consistent with the observed population (Figure 16). Future work will compare the characteristics of the NEOs to their probable source regions within the Main Belt and comets and will attempt to further refine the distribution of NEO orbital elements.

We finally note that it is possible that some short-arc NEOs submitted to the MPC can be classified as MBAs. It is possible to ascertain the fraction of objects that are misclassified by generating and sending a set of synthetic NEOs to the MPC in the form of tracklets (right ascensions, declinations, times, and spacecraft velocities). The digest score used to determine the probability that an object is an NEO could then be run on each synthetic tracklet to determine the fraction that would be erroneously classified as MBAs. This analysis will be the subject of future work in order to determine the fraction of misclassified NEOs likely to exist. At present, this fraction remains unquantified, and as stated above, the error estimates provided should be regarded as lower limits.

\subsection{NEOs As Future Targets for Exploration}

NEOs have been discussed as possible destinations for human exploration (Abell et al. 2009), and there has been a recent push to obtain characterization information for objects with low $\Delta v$ (Shoemaker \& Helin 1978), since these tend to require the least energy to reach. Objects with low $\Delta v$ are easier to reach with robotic missions as well. Figure 18 shows the albedo and size distributions for 80 NEOs that were observed by NEOWISE during the fully cryogenic portion of the mission that have $\Delta v<7 \mathrm{~km} \mathrm{~s}^{-1}$ as computed by Lance Benner's list of $\Delta v$ for all NEOs observational arcs longer than 0.1 years (this being a rough measure for how well an object's orbit is likely to be known; objects with observational arcs shorter than this will most likely be extremely difficult to locate in the future). Using a similar approach, Mueller et al. (2011) studied 65 NEOs with $\Delta v<7 \mathrm{~km} \mathrm{~s}^{-1}$; however, while there appear to be many targets, in practice, only a handful of NEOs will actually be suitably located in the timeframe of interest for human exploration (between 2020 and 2050). Among our detections of low $\Delta v$ NEOs, (3361) Orpheus and (207945) have close approaches in the next two decades; both have moderate to high albedos $(0.28 \pm 0.09$ and $0.16 \pm 0.02$, respectively) consistent with other S-complex objects observed by NEOWISE (M11D). NEOs with low albedos are potentially targets of greater interest, as these may possess volatile materials such as water that could be used as in situ resources for explorers. While one object among our sample (1996 GQ) has $p_{V}=0.02 \pm 0.002$ and $\Delta v \sim 6.5 \mathrm{~km} \mathrm{~s}^{-1}$, it may not approach Earth sufficiently closely to be readily accessible to human explorers in the 2025 timeframe.

Many of the NEOs being considered for human exploration have very large $H$ values, ranging as high as $H=27-28 \mathrm{mag}$ (Abell et al. 2009). As we have shown in earlier sections, NEO albedos range widely from $\sim 0.01$ to $\sim 0.6$ or higher. If only visible photometry is available, a proposed target with $H=27$ could be as small as only $5 \mathrm{~m}$. Such an object would be similar in size (or smaller) than a visiting crew capsule: a less-than-ideal target. It is important to provide solid diameter estimates for any potential target rather than relying solely upon $H$ due their widely varying albedos. By virtue of observing near $90^{\circ}$ solar elongation, NEOWISE was able to discover an unusual NEO during the post-cryogenic mission that may represent the first of a new class of objects well suited for human exploration. 2010 TK7 (Connors et al. 2011) is the first known Earth Trojan asteroid; although its inclination is too high to make its relative velocity low enough to be easily accessible, 

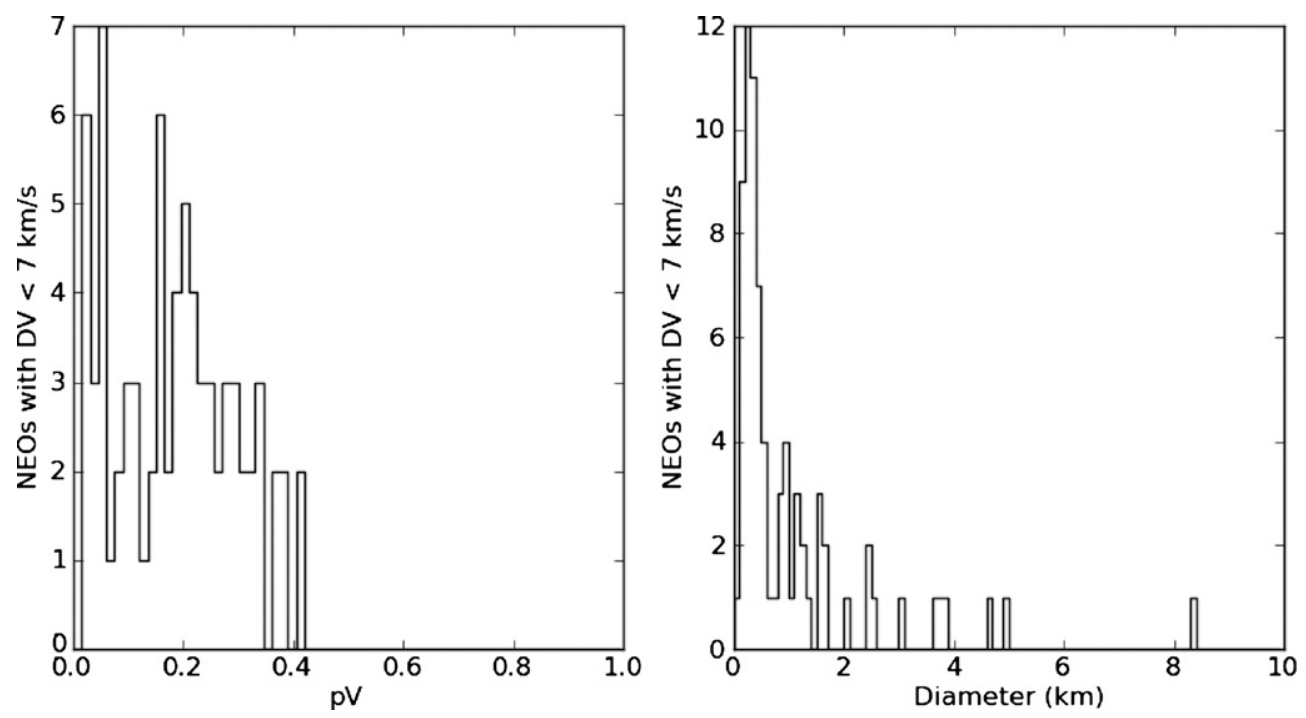

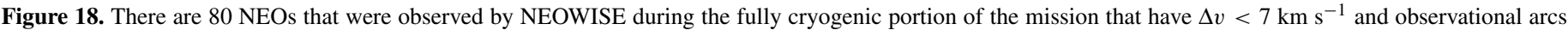

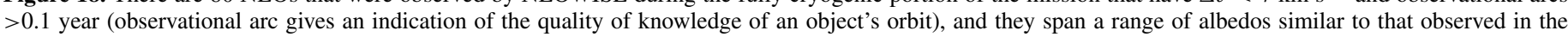

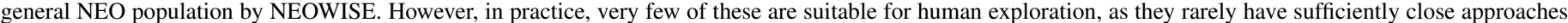
in the timeframe of interest (2020-2050).

similar objects in more energetically favorable Trojan orbits may exist.

\section{CONCLUSIONS}

The NEOWISE project has resulted in the acquisition of a sample of NEOs that is essentially unbiased with respect to visible albedo, allowing us to compute the numbers, sizes, and albedos of NEAs down to $\sim 100 \mathrm{~m}$ with reduced errors relative to previous work. By virtue of being in space, NEOWISE is characterized by its well-known sensitivity, number and location of pointings, and consistent image quality. We have shown that the Spaceguard goal of identifying $90 \%$ of all NEAs larger than $1 \mathrm{~km}$ has been met and exceeded. Furthermore, the number of NEAs with diameters as small as $100 \mathrm{~m}$ is likely to be less than previously suspected. We must apply similar analysis techniques to the subset of the NEAs that are potentially hazardous to determine whether this implies that the hazard is commensurately lower; this will be the subject of future work. We note that this analysis applies only to NEAs; a future work will assess the population of NECs, as their variable levels of activity can complicate efforts to obtain sizes and albedos for them (cf. Bauer et al. 2011a, 2011b).

Using the methods described above, we find that of the $\sim 8000$ NEOs known to date, $\sim 5200$ of them are larger than $100 \mathrm{~m}$. Since we estimate that there are 20,500 \pm 3000 NEAs in total with $D>100 \mathrm{~m}$, the $\sim 5200$ that have been found to date represent a relatively small fraction of the total that exist, implying that many still remain to be discovered. However, our sample contains only handful of objects smaller than $100 \mathrm{~m}$, so we are unable to confirm whether or not the cumulative size distribution slope remains the same below this limit. Although Harris (2008) found that the size distribution slope breaks at $D \sim 50-100 \mathrm{~m}$ and has a steeper slope below this limit, suggesting that the relative number of smaller objects is larger, we are unable to comment reliably on the numbers of NEAs below this size range with the existing NEOWISE sample at present.

Contrary to previous analyses with smaller data sets, we find no strong correlation of size with visible albedo. This result suggests that previous work (including those analyses attempting to link size and albedo to space weathering) has been hampered by observational biases, although the current lack of taxonomic classifications of most of the small NEOs observed by NEOWISE precludes a firm conclusion on this point (for a more extensive discussion of the links between size, albedo, and taxonomic classifications, see also Mainzer et al. 2011d). We note that the inclusion of the relatively small fraction of NEOWISE-discovered NEOs that did not receive visible followup is likely to increase the numbers of small, low albedo objects, further reducing the likelihood of a correlation between albedo and size. Compared with the average distribution of visible albedos found in the Main Belt (Masiero et al. 2011), the NEOs are preferentially brighter, following a roughly bimodal distribution with $\sim 40 \%$ having $p_{V}<0.1$. As expected given that we have shown that WISE is essentially unbiased with respect to $p_{V}$, NEOWISE preferentially discovered a higher fraction of low albedo NEOs than that found by visible light surveys, with $53 \%$ having $p_{V}<0.1$. With the improved number, size, and albedo distributions of the NEAs in hand, we can now begin to refine our understanding of their probable source regions within the Main Belt and the comets.

Our thermal models have revealed that the NEATM beaming parameter $\eta$ is correlated with phase angle/heliocentric distance, resulting from the fact that the temperature distribution as a function of latitude and longitude across an asteroid's surface is more complex than that assumed in the NEATM. Nevertheless, we have identified a number of NEOs with unusual properties such as (1865) Cerberus and NEOs with large-amplitude light curves; our understanding of these objects would benefit from continued study and additional follow-up observations. Similarly, the assumption that all objects can be reasonably represented as spherical should be revisited, and attempts should be made to determine three-dimensional shape models and rotational states.

We note that our results will be improved by the incorporation of the second generation of WISE data processing, as well by continued visible light follow-up of NEOWISE-discovered NEOs, both to improve their orbits and to refine their $H$ and $G$ values. The albedos that we have computed depend entirely on the quality of the $H$ and $G$ values that underpin them; while we have accounted for typical random errors associated with $H$ 
and $G$, if these parameters have systematic offsets or trends, these effects can change the computed albedos. Our understanding of the numbers, sizes, and albedos of the NEOs will be improved by detection of more objects in the WISE data set at lower signal-to-noise values as well as by mining the WISE data in order to compute diameters and albedos for previously known objects that can only be detected by combining all available images of them. We must also determine the debiased population statistics of the NECs and potentially hazardous objects in order to better constrain the characteristics of these populations; this will be the subject of future work. The fraction of NEOs that are not identified correctly by the MPC digest score remains unquantified at present. Nevertheless, even with these caveats, the NEOWISE portion of the WISE project has significantly improved our understanding of the NEAs and paves the way for future studies.

This publication makes use of data products from the Widefield Infrared Survey Explorer, which is a joint project of the University of California, Los Angeles, and the Jet Propulsion Laboratory/California Institute of Technology, funded by the National Aeronautics and Space Administration. This publication also makes use of data products from NEOWISE, which is a project of the Jet Propulsion Laboratory/California Institute of Technology, funded by the Planetary Science Division of the National Aeronautics and Space Administration. We thank our referee, Dr. Alan Harris of DLR, for his thoughtful comments which materially improved this work. We also thank Dr. Alan Harris of the Space Sciences Institute for useful conversations. We gratefully acknowledge the extraordinary services specific to NEOWISE contributed by the International Astronomical Union's Minor Planet Center, operated by the HarvardSmithsonian Center for Astrophysics, and the Central Bureau for Astronomical Telegrams, operated by Harvard University. We also thank the worldwide community of dedicated amateur and professional astronomers devoted to minor planet follow-up observations. This research has made use of the NASA/IPAC Infrared Science Archive, which is operated by the Jet Propulsion Laboratory, California Institute of Technology, under contract with the National Aeronautics and Space Administration.

\section{REFERENCES}

Abell, P., Korsmeyer, D., Landis, R., et al. 2009, Meteorit. Planet. Sci., 44, 1825 Alvarez, L., Alvarez, W., Asaro, F., \& Michel, H. 1980, Science, 208, 1095

Bauer, J. M., Mainzer, A. K., Grav, T., et al. 2011a, ApJ, submitted

Bauer, J. M., Walker, R. G., Mainzer, A. K., et al. 2011b, ApJ, 738, 171 Bhattacharya, B., Noriega-Crespo, A., Penprase, B., et al. 2010, ApJ, 720, 113 Boslough, M. B., \& Harris, A. W. 2008, Global Catastrophes in Perspective: Asteroid Impacts vs Climate Change, American Geophysical Union, Fall Meeting 2008, Abstract \#U41D-0034

Bottke, W., Morbidelli, A., Jedicke, R., et al. 2002, Icarus, 156, 399

Bowell, E., Hapke, B., Domingue, D., et al. 1989, in Asteroids II, ed. R. P. Binzel, T. Gehrels, \& M. S. Matthews (Tucson, AZ: Univ. Arizona Press), 524

Chapman, C. 2004, Earth Planet. Sci. Lett., 222,

Connors, M., Wiegert, P., \& Veillet, C. 2011, Nature, 475, 481

Cutri, R. M., Wright, E. L., Conrow, T., et al. 2011, Explanatory Supplement to the WISE Preliminary Data Release Products http://wise2.ipac.caltech.edu/ docs/release/prelim/expsup/wise_prelrel_toc.html

Delbó, M., Harris, A. W., Binzel, R. P., Pravec, P., \& Davies, J. K. 2003, Icarus, 166,116

Duncan, M., \& Levison, H. 1997, Science, 276, 1670

Gaffey, M., Cloutis, E. A., Kelley, M. S., \& Reed, K. L. 2002, in Asteroids III, ed. W. F. Bottke, A. Cellino, P. Paolicchi, \& R. P. Binzel (Tuscon, AZ: Univ. Arizona Press), 183

Grav, T., Jedicke, R., Denneau, L., et al. 2011, PASP, 123, 423

Harris, A. 1998, Icarus, 131, 291
Harris, A. 2008, Nature, 453, 1178

Harris, A., Mueller, M., Lisse, C., \& Cheng, A. 2009, Icarus, 199, 86

Harris, A. W. 2006, in Proc. 229th Symposium of the IAU on Asteroids, Comets, Meteors, Bzios, Rio de Janeiro, Brazil, 2005 August 7-12, ed. L. Daniela, M. Sylvio Ferraz, \& F. J. Angel (Cambridge: Cambridge Univ. Press), 449

Helin, E., Pravdo, S., Rabinowitz, D., \& Lawrence, K. 1997, in Proc. International Conference on Near-Earth Objects, the United Nations International Conference, New York, New York USA, 1995 April 24-26, ed. J. L. Remo (Annals of the New York Academy of Sciences, Vol. 822), 6

Hildebrand, A., Penfield, G., Kring, D., et al. 1991, Geology, 19, 867

Jedicke, R., Larsen, J., \& Spahr, T. 2002, in Asteroids III, ed. W. F. Bottke, A. Cellino, P. Paolicchi, \& R. P. Binzel (Tucson, AZ: Univ. Arizona Press)

Kaasalainen, M., Pravec, P., Krugly, Y., et al. 2004, Icarus, 167, 178

Koehn, B. W., \& Bowell, E. L. G. 2000, BAAS, 32, 1018

Kubica, J., Denneau, L., Grav, T., et al. 2007, Icarus, 189, 151

Larson, S. 2007, in IAU Symp. 236, Near Earth Objects, Our Celestial Neighbors: Opportunity and Risk, ed. G.B. Valsecchi, D. Vokrouhlicky, \& A. Milani (Cambridge: Cambridge Univ. Press), 323

Lebofsky, L., \& Spencer, J. 1989, Asteroids II (Tucson, AZ: Univ. Arizona Press), 128

Lebofsky, L., Veeder, G., Lebofsky, M., \& Matson, D. 1978, Icarus, 35, 336

Levison, H., \& Duncan, M. 1997, Icarus, 127, 13

Levison, H., Shoemaker, E., \& Shoemaker, C. 1997, Nature, 385, 42

Liu, F., Cutri, R., Greanias, G., et al. 2008, Proc. SPIE, 7017, 16

Luu, J., \& Jewitt, D. 1998, AJ, 98, 1905

Mainzer, A., Bauer, J., Grav, T., et al. 2011a, ApJ, 731, 53

Mainzer, A., Eisenhardt, P., Wright, E. L., et al. 2005, Proc. SPIE, 5899, 262

Mainzer, A., Grav, T., Masiero, J., et al. 2011b, ApJ, 736, 100

Mainzer, A., Grav, T., Masiero, J., et al. 2011c, ApJ, 737, L9

Mainzer, A., Grav, T., Masiero, J., et al. 2011d, ApJ, 741, 90

Mainzer, A., Masiero, J., Grav, T., et al. 2011e, ApJ, accepted

Masiero, J. R., Mainzer, A. K., Grav, T., et al. 2011, ApJ, 741, 68

Matson, D., (ed.) 1986, The IRAS Asteroid and Comet Survey (JPL D-3698; Pasadena, CA: JPL)

McMillan, R. S. 2007, in IAU Symp. 236, Near Earth Objects, Our Celestial Neighbors: Opportunity and Risk, ed. G. B. Valsecchi, D. Vokrouhlicky, \& A. Milani (Cambridge: Cambridge Univ. Press), 329

Morbidelli, A., \& Gladman, B. 1998, Meteorit. Planet. Sci., 33, 999

Morbidelli, A., Jedicke, R., Bottke, W., Michel, P., \& Tedesco, E. 2002, Icarus, 158,329

Morrison, D. 1992, The Spaceguard Survey: Report of the NASA International Near Earth Object Detection Workshop, QB651 N37 (Pasadena, CA: Jet Propulsion Laboratory/California Institute of Technology)

Mueller, M., Delbo, M., Hora, J., et al. 2011, AJ, 141, 109

National Research Council 2010, Defending Planet Earth: Near-Earth Object Surveys and Hazard Mitigation Strategies (Washington, DC: The National Academies Press)

Near-Earth Object Science Definition Team 2003, Study to Determine the Feasibility of Extending the Search for Near-Earth Objects to Smaller Limiting Diameters, National Aeronautics and Space Administration Office of Space Science Solar System Exploration Division, http://neo.jpl.nasa.gov/neo/report.html

Rabinowitz, D. 1997a, Icarus, 127, 33

Rabinowitz, D. 1997b, Icarus, 130, 287

Rabinowitz, D., Helin, E., Lawrence, K., \& Pravdo, S. 2000, Nature, 403, 165

Ryan, E., \& Ryan, W. 2008, in Proc. 2008 AMOS Technical Conference, ed. S. Ryan (The Maui Economic Development Board), E41

Shoemaker, E., Wolfe, R., \& Shoemaker, C. 1990, Geological Society of America Special Paper, 247

Shoemaker, E. M. 1983, Ann. Rev. Earth Planet Sci., 11, 461

Shoemaker, G., \& Helin, E. 1978, NASA, CP-2053, 245

Stokes, G., Evans, J., Viggh, H., Shelly, F., \& Pearce, E. 2000, Icarus, 148, 21 Stuart, J., \& Binzel, R. 2004, Icarus, 170, 295

Tedesco, E., Matson, D., Veeder, G., \& Lebofsky, L. 1988, in Proc. Third IRAS Conference on Comets to Cosmology, Lecture Notes in Physics, Vol. 297, ed. A. Lawrence (Berlin: Springer-Verlag), 19

Tedesco, E., Noah, P., Noah, M., \& Price, S. 2002, AJ, 123, 1056

Thomas, C., \& Binzel, R. 2010, Icarus, 205, 419

Trilling, D., Mueller, M., Hora, J. L., et al. 2010, AJ, 140, 770

Veeder, G., Hanner, M. S., Matson, D. L., et al. 1989, AJ, 97, 1211

Vernazza, P., Binzel, R., Thomas, C., et al. 2008, Nature, 454, 858

Warner, B., Harris, A., \& Pravec, P. 2009, Icarus, 202, 134

Weissman, P. 1996, in ASP Conf. Ser. 107, Completing the Inventory of the Solar System, ed. T. W. Rettig \& J. M. Hahn (San Francisco, CA: ASP), 265 Wetherill, G. W. 1988, Icarus, 76, 1

Wright, E. L., Eisenhardt, P. R. M., Mainzer, A. K., et al. 2010, AJ, 140, 1868

Wolters, S., Green, S., McBride, N., \& Davies, J. 2008, Icarus, 193, 535 